\title{
ILCEA
}

Revue de l'Institut des langues et cultures

d'Europe, Amérique, Afrique, Asie et Australie

$16 \mid 2012$

La culture progressiste à l'époque de la guerre froide

\section{Kreativität, morphologisches Weltbild und Erfindungsalgorithmus}

Zur transnationalen Emergenz von Kreativitäts- und Ideenfindungstechniken während der Nachkriegszeit und des Kalten Krieges

Morphological Thought and Invention Algorithms: On the transnational emergence of creativity and ideation techniques in the Cold War period

\section{Claudia Mareis}

\section{OpenEdition}

\section{Journals}

Édition électronique

URL : http://journals.openedition.org/ilcea/1260

DOI : $10.4000 /$ ilcea. 1260

ISSN : 2101-0609

Éditeur

UGA Éditions/Université Grenoble Alpes

Édition imprimée

ISBN : 978-2-84310-232-5

ISSN : 1639-6073

Référence électronique

Claudia Mareis, „Kreativität, morphologisches Weltbild und Erfindungsalgorithmus“, ILCEA [Online], 16। 2012, Online erschienen am: 04 Juli 2012, abgerufen am 22 März 2021. URL: http:// journals.openedition.org/ilcea/1260 ; DOI: https://doi.org/10.4000/ilcea.1260

Ce document a été généré automatiquement le 22 mars 2021.

(C) ILCEA 


\section{Kreativität, morphologisches Weltbild und \\ Erfindungsalgorithmus}

Zur transnationalen Emergenz von Kreativitäts- und Ideenfindungstechniken während der Nachkriegszeit und des Kalten Krieges

Morphological Thought and Invention Algorithms: On the transnational emergence of creativity and ideation techniques in the Cold War period

\section{Claudia Mareis}

1 Kreativitäts- und Ideenfindungstechniken ${ }^{1}$ sind symptomatische Produkte der Nachkriegszeit. Bekanntlich finden Heuristiken zur systematisch-operativen Ideengenese und Problemlösung bereits seit Jahrhunderten Verwendung, ${ }^{2}$ dennoch können die Nachkriegsjahre als ,eigentliche` Epoche der Kreativitätsförderung gelten; als eine Zeit, in der in ubiquitärer Weise eine Vielzahl von Kreativitätsratgebern und techniken lanciert und propagiert wurden. ${ }^{3}$ Bis in die Gegenwart stellen Techniken wie Brainstorming, Mind-Maps, Szenario-Analysen, Rollenspiele, morphologische Methoden oder systematische Erfindungsalgorithmen einen vitalen, wissenschaftlich jedoch wenig beachteten Bestandteil professioneller und populärer Wissensproduktion dar. ${ }^{4}$ Im Kontext von Ingenieursausbildungen Gestaltungskursen, Managementschulungen, Schreibseminaren und psychologischer Ratgeberliteratur erfreuen sich Kreativitätstechniken und die sie flankierende Rhetorik einer großen Popularität, gleichwohl übersteigt ihr methodischer Status selten den von unverbindlichen Inspirations- und Lockerungsübungen. ${ }^{5}$ Eine solche Sichtweise auf Kreativitäts-techniken blendet indes die beträchtlichen Anspannungen aus, unter sie denen nach dem Zweiten Weltkrieg und während des Kalten Krieges auf forcierte Weise entwickelt wurden.

2 Für die Frage nach einer ,fortschrittlichen Kultur ${ }^{\star}$ des Kalten Krieges sind Kreativitätstechniken in mindestens zweierlei Hinsicht aufschlussreich: Einerseits ist 
ihre interdisziplinäre, bisweilen antidisziplinäre (Pickering), anwendungsorientierte und technikaffine Ausrichtung charakteristisch für eine Wissenskultur der Nachkriegszeit, wie sie beispielsweise auch in der Kybernetik ${ }^{6}$ oder, institutionell betrachtet, im US-amerikanischen „military-industrial-academic complex “7 beobachtet werden kann. Andererseits ist die Entwicklung von Kreativitätstechniken in der Nachkriegszeit entgegen gängiger Annahmen nicht auf ein westlich-kapitalistisches Phänomen reduzierbar, sondern kann bis zu einem gewissen Maße auch in der Sowjetunion nachgezeichnet werden. Bei einer solchen transnationalenLesart wird gewahr, dass sich, obwohl die systematische Beförderung von Kreativität in Ost und West von jeweils unterschiedlichen ideologischen Narrativen gestützt wurde, in den entsprechenden Diskursen auch Gemeinsamkeiten und Analogien finden lassen. Seitens der USA wurde in der Nachkriegszeit besonders ein Mangel an innovativen Führungskräften als Motiv für die Erforschung und Beförderung von Kreativität angeführt. In der Sowjetunion prägte demgegenüber eine materialistisch-dialektische

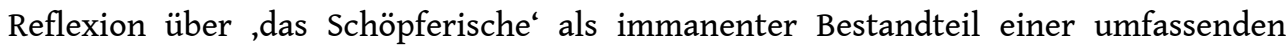
materialistischen Analyse von ,Arbeit' den Umgang mit Kreativität. Von beiden Seiten wurden indes auf ähnliche Weise radikale gesellschaftliche Veränderungen für einen veränderten Umgang mit der Kreativität verantwortlich gemacht. Die weitaus größten Gemeinsamkeiten finden sich dabei auf pragmatischer Ebene: bei den Kreativitätstechniken selbst, also in den konkreten Arten und Weisen, wie Kreativität während der Nachkriegszeit und des Kalten Krieges systematisch operationalisiert und ökonomisch nutzbar gemacht werden sollte.

3 Der vorliegende Text skizziert zunächst den Kontext und die Entstehung der Kreativitätsforschung in der Nachkriegszeit namentlich in den USA. Daran anschließend werden anhand der sogenannten morphologischen Methoden und der Theorie zur Lösung erfinderischer Probleme (TRIS) zwei Kreativitätstechniken aus den USA sowie der Sowjetunion exemplarisch vorgestellt. Diese Techniken werden vor dem Hintergrund von Kreativitätskonzepten und -ideologien der Nachkriegszeit und des Kalten Krieges diskutiert und punktuell miteinander in Beziehung gesetzt.

4 Vorwegnehmend kann festgehalten werden, dass Kreativitätstechniken während der Nachkriegszeit und des Kalten Krieges sowohl in kapitalistischen als auch sozialistischen Systemen ein produktives, dabei aber nicht unumstrittenes methodisches Repertoire bereitstellten, das versprach, technologischwissenschaftlichen Fortschritt auf systematische Weise zu produzieren. In dem von Angst ${ }^{8}$ getriebenen Wettkampf um militärisch-technologischen Vorsprung im OstWest-Konflikt galt Kreativität - und methodisch operationalisierte Kreativität zumal als Chance, essentieller noch als „Überlebenschance“.9

\section{Mit Kreativitätstechniken das Undenkbare denken}

Nach dem Zweiten Weltkrieg emergierte die Kreativitätsforschung in den USA als ein inter- oder bisweilen sogar antidisziplinäres Projekt über verschiedenste Wissensräume hinweg. Relevante Arbeiten zur Psychologie der Kreativität entstanden vor dem Hintergrund militärisch-defensiver Belange, so unterstützten beispielsweise die Atomenergie-Kommission (AEC) sowie die Nationale Wissenschaftsförderung (NSF) psychologische Kreativitätsforschung als einen festen Bestandteil in ihren Forschungsprogrammen. ${ }^{10}$ Kreativitätsforschung war indes nicht nur ein akademisches 
Projekt, das an den Universitäten angesiedelt war, sondern sie fand vor allem auch in praxis- und wirtschaftsnahen Kontexten statt. Sie wurde gleichermaßen von Militärstrategen, Futurologen, Psychologen, Ingenieuren, Designern, Managern oder Pädagogen betrieben. Im Hinblick auf eine historische und epistemische Bestimmung ist damit auch gesagt, dass Kreativitätstechniken keineswegs isoliert betrachtet werden können, sondern dass sie Bestandteil eines weitaus umfassenderen „Dispositivs der Kreativität" ${ }^{\text {"11 }}$ sind, das sich in der Nachkriegszeit durch das Ineinandergreifen und Vermischen von psychologischen, politischen, ökonomischen und wissenschaftlichen Bestrebungen konstituierte und sich durch eigens entworfene Heuristiken reifizierte.

6 Vermittels Szenario-Analysen, Rollenspielen oder moderierten BrainstormingSitzungen wurden selbst vermeintlich undenkbare Zukunftsszenarien wie ein drohender nuklearer Krieg tentativ ,durchgespielt' und strategische Planung sowie Handlungsfähigkeit konnte so, aller Unwägbarkeiten zum Trotz, zumindest auf virtueller Ebene weiterhin demonstriert werden. Laut Eva Horn sind derartige „Kriegsspiele“ als „heuristische Fiktionen von Freund und Feind“" zu verstehen, die der gefahrlosen Virtualisierung einer potentiellen Krisenoperation dienen: die „Logik einer Strategie“ kann in Kriegsspielen erprobt werden, „ohne ihre Folgen zu tragen“ ${ }^{12}$ Der namentlich im Brainstorming angestrebte Modus eines kritikfreien und damit vermeintlich schrankenlosen Denkens sowie der Anspruch, eine möglichst hohe Quantität an Ideen zu erzeugen, entsprach diesem Ansinnen in perfekter Weise. ${ }^{13}$

7 Als prominenter Anwender von Kreativitätstechniken in diesem Kontext gilt der Futurologe und Militärstratege Herman Kahn. Kahns Bestrebungen, Kreativität und Analyse zu verbinden, wurden von Donald Rumsfeld, ehemaliger Verteidigungsminister der Vereinigten Staaten, mit den Worten gewürdigt: „He boldly confronted public issues with creativity and the conviction [...] that thought and analysis could help make ours a better world.“ ${ }^{{ }^{14}}$ Eben diese vermeintlich gegenstrebige Kombination von Kreativität und Analyse, von Intuition und Planung bildeten die Basis für Kahns kontrovers diskutierte nukleare Szenarien. ${ }^{15}$

8 Seine Karriere begann Kahn in den späten 1940er Jahren als Physiker und Mathematiker bei der regierungsnahen RAND Corporation, ${ }^{16} 1961$ wechselte er zu dem von ihm gegründeten Hudson Institute. In den legendenumwobenen Think-Tanks beider Institute kreierte er einen ganzen „Haushalt von Medien, Methoden, Kreativitätstechniken“, der „das vielbeschworene Denken des Undenkbaren erst gewährte und bestimmte “. ${ }^{17} \mathrm{Er}$ war nachhaltig an den ,praktischen, epistemischen und methodologischen Problemen“ interessiert, die der „Umgang mit der Zukunft" einforderte ${ }^{18}$ und erprobte dieses Interesse auf unkonventionelle Weise. Die in den ,Denkfabriken' des Kalten Krieges angewandten Raumkonzepte, Sitzungsmodalitäten, Methoden und technischen Medien galten als neuartig und experimentell für ihre Zeit: „Spieltheorie, Operations Research und Systems Analysis [wurden] kombiniert mit Kreativitätstechniken [...] und appliziert mittels Szenario-Building, Folienprojektionen und (Computer-)Simulationsverfahren." ${ }^{19}$ Nicht zuletzt vermittelten sie eine gewisse ,Lässigkeit` im Umgang mit möglicherweise folgenschweren Problemlösungsstrategien. 20

9 Den innovativen Techniken lag freilich auch ein verändertes Bild von Wissensproduktion zugrunde, in dem Wissen nicht mehr als das „Verwaltungswissen“ eines Ist-Soll-Zustandes verstanden wurde, sondern als ein „Möglichkeitswissen“, in dem nicht das Naheliegende, sondern „im Gegenteil, das Undenkbare“ im Zentrum 
stand. ${ }^{21}$ Entsprechend der heterogenen, nach Albert Wohlstetter „pan-heuristischen “ ${ }^{22}$ Denk-, Planungs- und Ideenfindungsmethoden, die in den regierungsnahen ThinkTanks zur Anwendung kamen sowie aufgrund der unterschiedlichen disziplinären Herkünfte der Mitarbeitenden war die vorherrschende Wissenskultur von Interdisziplinarität und Vermischung geprägt. ${ }^{23}$ Der RAND-Mitarbeiter Robert D. Specht erinnert sich:

[The] compartments into which RAND is organized are not to be taken too literally. On the Engineering Division roster one will find the occasional political scientist, astronomer, physiologist, or psychologist. The Mathematics Division is headed by an astronomermathematician and uses the skills of the sociologist and the philosopher. Mathematicians, in turn, have infiltrated most Divisions. ${ }^{24}$

10 Seine Aussage vermag die Diversität disziplinärer, professioneller und methodologischer Anschauungen und Vorgehensweisen zu veranschaulichen, die für den Umgang mit einer ungewiss erscheinenden Zukunft im Kalten Krieg von Nöten schienen. Deutlich wird indes auch, dass der Schwerpunkt der genannten interdisziplinären Vermischung im Grunde aber immer noch stark auf einer naturwissenschaftlich-technischen Wissensbasis beruhte. Ron Robin konstatiert, dass sich das universitäre Fächerangebot „auf verzerrte Weise“ in den Denkfabriken niederschlug, insbesondere wurden Vertreter der geisteswissenschaftlichen Fächer marginalisiert: „Den Maßstab setzten die Naturwissenschaften [...]. Auch Fächer, die dem Selbstverständnis der Naturwissenschaften durch statistische Qualitätskontrollen und quantitative empirische Studien genügen konnten, hatten von Anfang an prominenten Anteil am Geschäft der Think Tanks. ${ }^{\text {“25 }}$ Bezeichnend für diese Entwicklung konstatierte der Medientheoretiker Marshall McLuhan in den 1960er Jahren: „The improvement of intuition is a highly technical matter.“ ${ }^{26}$

\section{Psychologische Kreativitätsforschung in den USA}

Die Beförderung von Kreativität in der Nachkriegszeit ist grundlegend auch als ein psychologisch-psychometrisches Projekt anzusehen. In den USA wurde das Interesse an Kreativität zu dieser Zeit entscheidend durch militärerfahrene Psychologen stimuliert. Sie sahen in der Kreativitätsforschung eine rentable Fortsetzung und Erweiterung der traditionellen psychometrischen Intelligenzforschung, etwa den Army Mental Tests, die während der beiden Weltkriege ausgiebig zur Selektierung von Armeeangehörigen erprobt und ausgereizt wurde. ${ }^{27}$ Dieser Tradition gemäß entwickelten sich die Verfahren, mit denen kreative Personen in der Nachkriegszeit identifiziert werden sollten, aus jenen psychometrischen Verfahren heraus, mit denen während Kriegszeiten potentielle Offiziere, Flugzeugmechaniker oder Atomwissenschaftler getestet und eingeschätzt wurden. ${ }^{28}$ Nur vorgeblich wandelte sich in der Nachkriegszeit und im Kalten Krieg auch das „Zielpublikum“, das im Visier der Kreativitätsforschenden stand. Zwar wurden verstärkt zivile und wirtschaftliche Interessen akzentuiert, gleichwohl blieb die Idee bestehen, dass durch gezielte psychometrische Erhebungen potentielle Leistungsträger effizient aus der Menge des ,Menschenmaterials` herausgefiltert werden konnten. Diese letztlich militärischstrategische Fundierung von Kreativitätsforschung führte dazu, dass der Kreativitätsbegriff von Zeitgenossen stets ambivalent rezipiert wurde. Kritiker der zeitgenössischen Intelligenzforschung monierten, dass der Interessenwandel von Intelligenz hin zu Kreativität vor allem „praktisch motiviert“ sei und dass, namentlich 
vor dem Hintergrund des Sputnik-Schocks, „der Wettkampf zwischen den USA und der UdSSR [...] eine nicht zu geringe Rolle" spiele. ${ }^{29}$ Auch die deutsche Literaturwissenschaftlerin Mechthild Curtis konstatierte 1976 mit kritischem Unterton:

Der Begriff [Kreativität] ist mit Vorsicht zu verwenden, nachdem er in den letzten zwei Jahrzehnten gerade von solchen US-Forschern populär gemacht wurde, die, durch die Air-Force-Flügel des Sputnikschocks beschwingt, von Wirtschaft und Militär finanziert, das humane Ziel verfolgen, mit Tests und anderen Verfahren zur Messung von schöpferischer Leistung möglichst potente Bombenbauer auszumachen [...]..$^{30}$

121950 plädierte der Präsident der American Psychological Association, der angesehene Psychologe und Intelligenzforscher Joy Guilford, mit Nachdruck für die Erforschung und Beförderung von Kreativität. ${ }^{31}$ Seine Rede gilt vielen bis heute als ein Schlüsseltext dieses Forschungsfeldes. Guilford, der während des Zweiten Weltkrieges selbst bei der Luftwaffe psychologische Auswahlverfahren für Piloten, Bombenschützen und Navigatoren durchführte, ${ }^{32}$ bemängelte in seinem Vortrag, dass die systematische Erforschung und Beförderung von Kreativität allzu lange vernachlässigt worden sei und dass nun ein großer Bedarf an innovativen, zukunftsfähigen Führungskräften in Wirtschaft und Militär bestehe. ${ }^{33}$ Mit dem Projekt ,Kreativitätsforschung ' stellte Guilford einen schier unerschöpflichen (da hochkomplexen) neuen Forschungsgegenstand in Aussicht, der nicht nur allergrößte methodische und epistemische Anstrengungen einforderte, sondern einen ebenso großen gesellschaftlichen, vor allem aber wirtschaftlichen Nutzen versprach. ${ }^{34}$

13 Aus methodischer Sicht wurde als besondere Schwierigkeit die wissenschaftliche Quantifizierbarkeit von kreativen Leistungen akzentuiert. ${ }^{35}$ Anders als die gängige Praxis der Intelligenzmessung es zu der Zeit vorschlug, schien die Erforschung von Kreativität nach einer über diskrete intelligible Fähigkeiten und Fertigkeiten hinausgehenden Betrachtungsweise der gesamten Persönlichkeits- und Charakterstruktur zu verlangen. Guilford konstatierte, dass das große „Problem des Psychologen [...] das der schöpferischen Persönlichkeit" sei. ${ }^{36} \mathrm{Zu}$ ergänzen ist indes, dass sich dieser Fokus auf die Persönlichkeit weniger der romantischen Idee eines einzigartigen künstlerischen Genies verpflichtet fühlte, sondern sich im Gegenteil überindividuell auf der Basis einer statistischen „Normalverteilung ${ }^{{ }^{37} 7}$ finden sollte. Anders als Genialität, die als exklusiv, bisweilen sogar als krankhaft galt und außerhalb gesellschaftlicher Normen stand, wurde Kreativität von Psychologen wie Guilford als basale menschliche Eigenschaft definiert, die jeder besaß - wenngleich sie in unterschiedlichen Ausprägungen vorkam. ${ }^{38}$ Pointiert gesagt: Genial waren nur wenige kreativ sein konnte, oder vielmehr sollte jeder sein. Jamie Cohen-Cole konkludiert in seiner profunden Studie über die Kreativitätsforschung in der US-Nachkriegszeit:

What we see in the way postwar Americans consistently marginalized genius is how very important it was to them that positive mental traits be adaptable to society. They consequently pictured creativity as a social affair. While numerous publications examined the kinds of environments that would foster creativity, there was almost no literature on its genetic basis. Many psychologists, cultural critics, and educators agreed that creativity was much more a product of nurture than of nature. Accordingly, educational experts filled their journals with articles on how to promote student creativity. ${ }^{39}$

14 Vermittels einer solchen Definition wurde Kreativität von einem raren Gut zu einer weitverbreiteten wirtschaftlichen Ressource umdeklariert. Sie wurde dem Bereich des abnormalen, pathologischen Handelns entrissen und für die Zwecke einer „verwalteten Welt $^{440}$ (Adorno) normiert - ohne dass man dabei jedoch vollends auf genialische 
Attribute verzichten wollte. Ein Kreativitätsratgeber der Zeit resümiert, dass „der Kreative“ stets zwei Leben führe: ein „wildzerrauftes genialisches“ und eine „bürokratische Existenz". ${ }^{41} \mathrm{Er}$ stellte dergestalt eine idealtypische Mittlerfigur zwischen dem „konservativem Bürokraten“ und dem „revolutionärem Chaoten“ dar beides Charaktertypen, die in der westlichen Nachkriegswelt aufgrund ihrer Nähe zum Faschismus einerseits sowie zum Sozialismus andererseits als suspekt galten. Kreativ zu sein, statt blindlings vorgegebenen Ideen zu folgen, oder im Gegenteil, statt planlos zu rebellieren, schien vor dem Hintergrund der Ideologien des Zweiten Weltkrieges beziehungsweise des Kalten Krieges ein erstrebenswertes Ideal zu sein.

Der Typus eines liberalen, undogmatisch denkenden, also kreativen (notabene westlichen) Intellektuellen oder Unternehmers wurde mithin jenem Charaktertypus gegenübergestellt, den die Berkeley-Gruppe um Theodor W. Adorno und Else FrenkelBrunswik 1950 in ihrer Faschismus-Studie als "autoritäre Persönlichkeit“ identifizierten..$^{42}$ Gemäß den Autoren von The Authoritarian Personality waren demokratische gesinnte Personen zu mehr Kreativität, Spontaneität, Imagination und Selbstaktualisierung fähig als solche mit einem autoritären Charakter, entsprechend wurde Kreativität selbst als wesentliche Eigenschaft für die Demokratie angesehen. ${ }^{43}$ Obschon die kreative Persönlichkeit also als Gegenstück zur autoritären entworfen wurde, wurde ihr keineswegs ein durchschlagendes revolutionäres Potential zugebilligt. Die gesuchte Kreativität sollte eine systemstabilisierende, keine „gesellschaftsverändernde Kraft sein“". ${ }^{4}$

Flankierend zu dieser Charakterisierung von Kreativität wurde eine wechselwirksame Simultanisierung individueller Schöpfungskraft und kollektiver Produktionskräfte eingeleitet, die der Soziologe Ulrich Bröckling mit den Worten kommentiert: „Was die Gesellschaft als Ganze benötigte, sollte zugleich das sein, worin die Einzelnen sich selbst finden. “" ${ }^{45}$ Das zu stimulierende kreative Potenzial des Einzelnen sollte möglichst passgenau in die Kollektivform von sowohl demokratischer Gesinnung als auch kapitalistischen Produktionsmitteln eingemündet werden. Kreativität wurde dabei nicht einfach nur als ein mentaler Prozess verstanden, sondern als eine nützliche und produktive soziale Eigenschaft, die mithin an den aus ihr resultierenden Produkten gemessen wurde: „whether they were poems, patents, buildings, or bombs. ${ }^{\text {" }}{ }^{6}$

Zum Instrumentarium des Projekts ,Kreativitätsforschung' gehörte - nebst einem rhetorischen Argumentationsspektrum - auch eine ganze Palette medial-materialer beziehungsweise performativer Instrumente, wie etwa multidimensionale Kreativitätsskalen, psychometrische Kreativitätstests sowie eine Vielzahl von anwendungsorientierten Kreativitätstechniken und -trainings. Kritisch anzumerken ist diesbezüglich, dass die Basis jenes psychologischen Instrumentariums, das Kreativität wissenschaftlich messen und befördern sollten, sich meist aus populären, nichtwissenschaftlichen Annahmen zum ,Wesen' und Auftreten von Kreativität zusammensetzte. Beispielsweise bezogen Kreativitätspsychologen sich oft unkritisch auf die Selbstaussagen von Künstlern und Wissenschaftlern zu ihren Arbeitsprozessen, oder herausragende, als besonders kreativ geltende Personen, wie etwa Albert Einstein, wurden zu heroisierten Vorbilder für die Herausarbeitung kreativer Eigenschaften stilisiert. ${ }^{47}$ Cohen-Cole kommentiert diese populärwissenschaftlichen Grundlagen der Kreativitätsforschung auf kritische Weise:

[...] psychologists devised instruments for measuring and understanding creativity that were calibrated by preexisting nonscientific notions of what it was and who possessed it. That is, they found exemplary individuals who were already known for their creativity and 
then built tools that could distinguish these people from everyone else. Psychologists thereby constructed psychological theory directly on top of a foundation of popular wisdom about creativity. Akteuren, Disziplinen, Institutionen, Interessen, Medien und Praktiken eine gezielt koordinierte und konsequent durchgeführte Aktion zur Kreativitätsförderung in der Nachkriegszeit darstellen würde. Die zahlreichen und im vorliegenden Text auch nicht hinreichend aufgeführten Einflüsse und Vernetzungen sind letztlich zu komplex und ihr historisches Zusammenspiel schlichtweg zu kontingent, als dass eine derart kausalistische Lesart von Kreativitätsforschung möglich und sinnvoll wäre. Gleichwohl liegt - nicht zuletzt vor dem Hintergrund der namhaften Unterstützung der Kreativitätsforschung durch die US-amerikanische Regierung - die Lesart nahe, dass der Entwurf einer antiautoritären, flexiblen, aber zugleich systemstabilisierenden kreativen Persönlichkeit in der Nachkriegszeit einen weitreichenden Versuch darstellte, ,ungezähmte individuelle Schöpfungs- und Arbeitskraft für standarisierte Produktions- und Bürokratisierungsprozesse zu erschließen und gemäß hegemonialer Interessen seitens von Staat und Wirtschaft zu verfestigen.

In dieser Lesart gewinnt auch der Gedanke an Bedeutung, dass das Interesse an Kreativität in der Nachkriegszeit eine übergeordnete kollektivpsychologische Dimension besaß, die ganze Weltbilder und Systemideologien umfasste, entsprechend derer das einzelne Individuum ausgerichtet und normiert werden sollte. Zugleich aber und scheinbar im Gegensatz dazu, eröffneten die zahlreich publizierten Kreativitätsratgeber in der Nachkriegszeit dem Einzelnen zumindest in begrenztem Rahmen doch die Möglichkeit, festgefahrene Denk- und Handlungsmuster zu durchbrechen und so vielleicht wieder neue Perspektiven für ein kriegsbeschädigtes Leben zurückzuerlangen. Kreativitätstechniken wären in einer solchen, positiv emanzipatorisch gewandten Lesart nicht bloß als Produktionsmittel zu verstehen, sondern sind dem verwandt, was Michel Foucault „Technologien des Selbst“ nannte: Technologien, die „instrumentell der Herausbildung eines neuen Selbst dienstbar gemacht werden ". ${ }^{48}$ In diesem Sinne versprach auch Alex Osborn, der ,Erfinder' der Brainstorming-Technik, in seinen auflagenstarken Kreativitätsratgebern der 1950er und - 60 Jahre, dass erst ein richtig geschultes und genutztes Imaginationsvermögen zu einem gelingenden Privat- und Geschäftsleben verhelfen könne. Richtig angewandte Imagination versprach, selbst die kühnsten Träume wahr werden zu lassen: „[to] turn your day dreams into reality. “49

\section{Methodisches Denken im morphologischen Weltbild}

Um den bis hierhin umrissenen Gegenstand der westlichen Kreativitätsforschung in der Nachkriegsära weiter zu konkretisieren und in seiner Pragmatik zu verdeutlichen, soll im Folgenden exemplarisch auf eine Kreativitätstechnik näher eingegangen werden. Es handelt sich dabei um die sogenannten morphologischen Methoden, bestehend aus einem ganzen Set von Problemlösungs-, Denk- und Ideenfindungstechniken. Diese sind ihrerseits eingebettet in ein systematisch-holistisches Weltbild, das deutlich die diskursiven Prägungen der Nachkriegszeit und des Kalten Krieges aufweist. ${ }^{50}$

Entwickelt wurden die Methoden von dem Schweizer Astrophysiker Fritz Zwicky (1898-1974), der nach einem Physik-Studium an der Eidgenössisch Technischen

ILCEA, 16 | 2012 
Hochschule in Zürich in die USA emigrierte und dort von 1942 bis 1968 als Professor für Astrophysik am California Institute of Technology in Pasadena lehrte. Obwohl er mit seiner direkten, manchmal undiplomatischen Art und seinen unkonventionellen Ideen oft aneckte, genoss er als Astrophysiker ein beachtliches internationales Renommee. ${ }^{51}$ Neben der Astrophysik verfolgte er eine ganze Reihe von weiteren Interessen und Tätigkeiten, so war er erfolgreich als Astronom, als Entwickler von Raketentriebwerken und Antriebsstoffen sowie, während des Zweiten Weltkrieges, als militärischer Berater für die US-amerikanische Regierung tätig. ${ }^{52}$ In seinen Selbstdarstellungen schrieb Zwicky seine wissenschaftlichen und professionellen Erfolge stets einem konsequenten morphologischen Denken und Handeln $\mathrm{zu}$ - wenngleich gerade seine Beschäftigung mit der Morphologie seitens von Wissenschaftskollegen oft kontrovers aufgenommen wurde. ${ }^{53}$ Zur Präzisierung sollte an dieser Stelle angefügt werden, dass Zwicky selbst auf die zeitgenössische Kreativitätsforschung und auf andere Kreativitätstechniken selten Bezug nahm. ${ }^{54}$ Jedoch weisen seine Arbeiten zahlreiche diskursive Analogien dazu auf. Ebenfalls - und das ist für das Thema des vorliegenden Textes relevant wurden seine Methoden noch zu seinen Lebzeiten und mit seinem Wissen von anderen ,Morphologen“ in ein allgemeineres Inventar von Kreativitätstechniken eingefügt und als solche behandelt..$^{55}$

Der Ausdruck ,Morphologie‘, den Zwicky als programmatische Chiffre für seinen Denkansatz und seine Methoden verwendete, lehnte er (allerdings nur vage) an das Morphologie-Konzept von Johann Wolfgang von Goethe an. ${ }^{56}$ Goethe konzipierte die Morphologie anfangs des 19.Jahrhundert als eine ganzheitlich-teleologische Betrachtung von organischen Prozessen (namentlich bei der Pflanzengenese). ${ }^{57}$ Für Zwicky waren indes „nicht nur geometrische, geologische oder biologische Formen und ihre gegenseitigen Verhältnisse“ von Interesse, sondern „die strukturellen Beziehungen zwischen Phänomenen, Handlungen und Ideen jeglicher Art " ${ }^{\text {" }}{ }^{5}$ In diesem Sinne erweiterte er den goetheschen Ansatz von der organischen Welt auf sämtliche nur vorstellbaren Frage- und Problemstellungen (physikalische, technische, kulturelle, moralische, etc.) und interpretierte ihn in methodischer Hinsicht als einen generalistischen Modus des „strukturierten Denkens“. ${ }^{59}$ Morphologie war für Zwicky schlichtweg eine „Totalitätsforschung“ oder "total thinking“, ${ }^{60}$ entsprechend interessierten ihn in sehr umfassender, allgemeiner Weise „Wesen und Wandel materieller und geistiger struktureller Zusammenhänge“ ${ }^{61}$

Mit der Entwicklung von systematischen Ideenfindungstechniken und Denkmethoden beschäftigte sich Zwicky bereits während seiner Jugendjahre in der Schweiz, beispielsweise zu Fragen der Stenographie oder des Bergsteigens. ${ }^{62}$ Vermutet wird, dass Zwicky seinen morphologischen Denkansatz vor dem Hintergrund zeithistorischer Politikdiskussionen als eine liberale, antikommunistische Gegenposition formulierte, insbesondere in Abgrenzung zu dem etwa zeitgleich wie Zwicky in Zürich weilenden Lenin. ${ }^{63}$ Während des Zweiten Weltkrieges nutzte Zwicky, nunmehr in den USA wohnhaft, die Gelegenheit weitere morphologische Analysen im Bereich des Zivilschutzes auszutesten. ${ }^{64}$ Nach dem Zweiten Weltkrieg begann er dann die Morphologie konsequent im Rahmen von akademischen Vorträge und Texte anzuwenden und $\mathrm{zu}$ verbreiten - wobei ihn entsprechend seiner vielen Interessen sowohl wissenschaftlich-technische als auch gesellschaftliche Fragestellungen beschäftigten. ${ }^{65}$ Wie bereits angedeutet, wurde seine Beschäftigung mit der Morphologie von Fachkollegen sehr unterschiedlich bewertet. Manchen galten seine morphologischen Methoden als $\mathrm{zu}$ universalistisch und unwissenschaftlich 
(„subjektivistisch“).66 Für andere jedoch, wie für den Astrophysiker und Wissenschaftsforscher Erich Jantsch, stellten sie wegweisende Methoden der Zukunftsforschung und des Technological Forecasting dar. ${ }^{67}$

Fritz Zwicky selbst setzte sich, ungeachtet der Kritik an seinem Werk, zeitlebens für die „praktische Anwendung ${ }^{{ }^{\prime \prime} 68}$ von wissenschaftlich fundierten Methoden ein, die zur "Systematisierung der Erfindungsgabe“ führen sollten. ${ }^{69}$ Erfindungen und Entdeckungen aller Art sollten damit, so seine Hoffnung, ,auf eine methodische Art“ produziert werden, die „Intuition“ sollte befördert werden, ohne dass man sich nur „aufs Pröbeln oder den Zufall“ verlassen musste. ${ }^{70}$ Wegweisend in seinen Bestrebungen war für ihn der Aspekt der Vorurteilslosigkeit. Durch eine wissenschaftliche - sprich: operationalisierte, wiederholbare und intersubjektiv nachvollziehbare - methodische Vorgehensweise wollte Zwicky ergebnisoffene und vermeintlich vorurteilsfreie Problemlösungen und Ideenfindungen befördern.

Das Spektrum der morphologischen Methoden wuchs im Verlauf der Jahrzehnte kontinuierlich an und umfasst beispielsweise die „Methode der Systematischen Feldüberdeckung“, die „Methode der Gerichteten Intuition“, die „Methode der Negation und Konstruktion“, die "Methode der Extreme“, die „Methode des Systematischen und positiven Einsatzes von Unvollkommenheiten“ oder die "Methode des Morphologischen Kastens s". ${ }^{71}$ Für viele der genannten Methoden kann in Anschlag gebracht werden, dass sie keineswegs neu sind, sondern sich auf bekannte, historisch tradierte kombinatorisch-logische Verfahren stützen, wie beispielsweise auf die mittelalterliche Kombinatorik des Ramon Llull. ${ }^{72}$ Ungeachtet dieser Tatsache gelang es Zwicky jedoch, diese Methoden für sich $\mathrm{zu}$ beanspruchen und ihnen in der Gesamtschau seines „morphologischen Weltbildes“einen prominenten Stellenwert zu geben.

Abb. 1. und 2. - Der Morphologische Kasten dargestellt in zwei bzw. drei Dimensionen

\section{Der Morphologische Kasten der Verbaltensformen}

\begin{tabular}{|c|c|c|c|}
\hline Hauptparameter & \multicolumn{3}{|c|}{ Komponenten der Parameter } \\
\hline$P_{1}=\begin{array}{l}\text { Art des } \\
\text { Vorgehens }\end{array}$ & $\begin{array}{c}\mathrm{P}_{11} \\
\text { diktatorisch }\end{array}$ & $\begin{array}{c}P_{12} \\
\text { gegenseitiges } \\
\text { Einver- } \\
\text { ständnis }\end{array}$ & $\begin{array}{c}\mathrm{P}_{13} \\
\text { absolut } \\
\text { objektiv }\end{array}$ \\
\hline $\begin{aligned} P_{2}= & \text { Qualitativer } \\
& \text { Charakter } \\
& \text { des } \\
& \text { Standards }\end{aligned}$ & $\begin{array}{c}\text { P21 }_{21} \\
\text { Körper } \\
\text { belebt oder } \\
\text { leblos }\end{array}$ & $\begin{array}{c}\mathrm{P}_{22} \\
\text { Erscheinung }\end{array}$ & $\begin{array}{c}\mathrm{P}_{23} \\
\text { Begriff }\end{array}$ \\
\hline $\begin{aligned} P_{3}= & \text { Folgen der } \\
& \text { Abweichung } \\
& \text { vom Verhal- } \\
& \text { tenskodex }\end{aligned}$ & $\begin{array}{c}P_{31} \\
\text { keine }\end{array}$ & $\begin{array}{c}\mathrm{P}_{32} \\
\text { destruktiv } \\
\text { (Bestrafung) }\end{array}$ & $\begin{array}{c}\text { P }_{33} \\
\text { konstruktiv } \\
\text { (Belohnung) }\end{array}$ \\
\hline
\end{tabular}




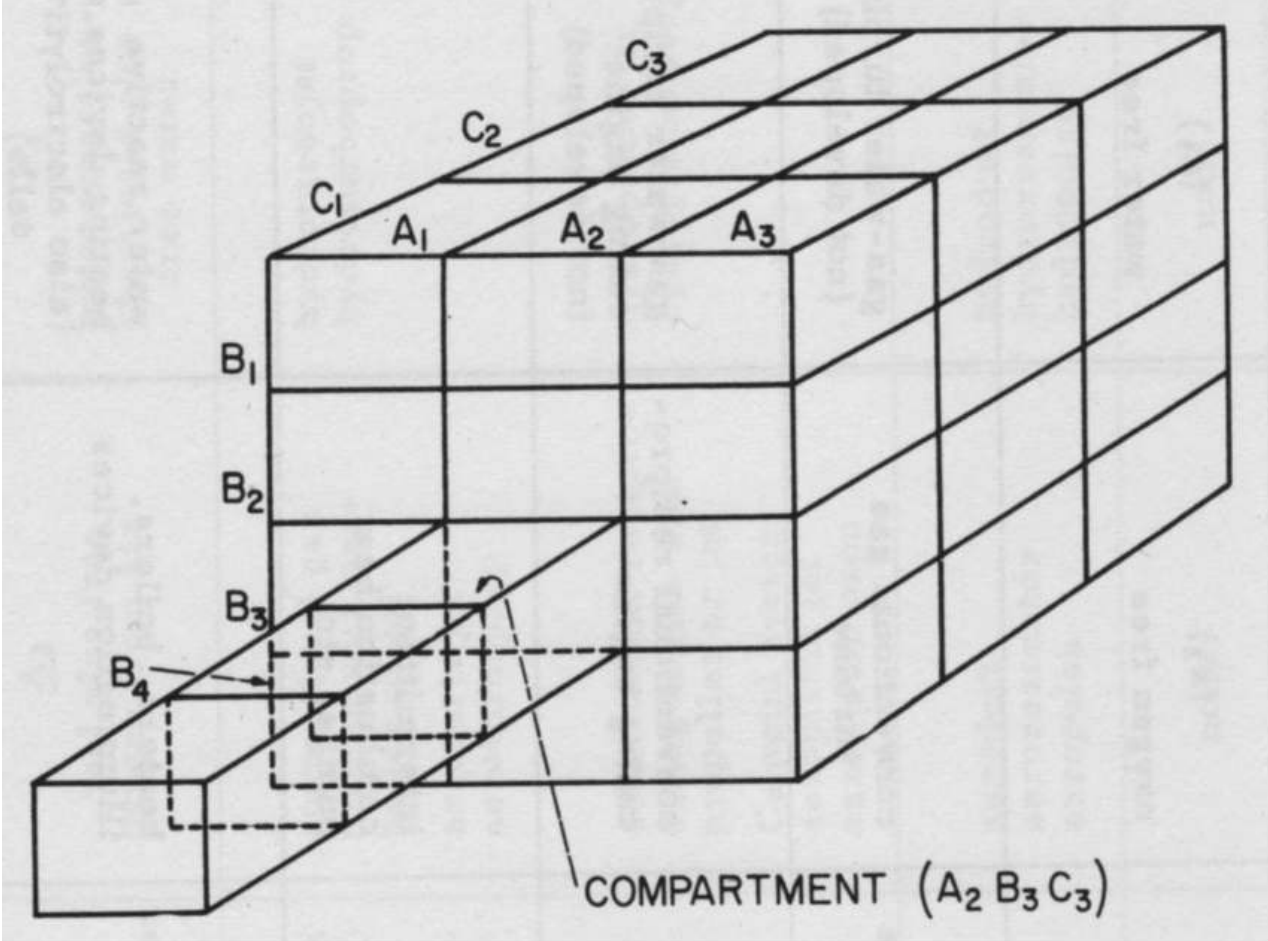

Das Herzstück seiner Methodologie bildet der bis heute einschlägig bekannte „morphologische Kasten“. [Abb. 1 und 2] Anders als der Name suggeriert, ist der morphologische Kasten kein reales dreidimensionales objekt, sondern ein Diagramm. Genauer gesagt, handelt es sich dabei um eine kombinatorische Matrix, die in Form einer Tabelle oder eines Würfels aufgezeichnet werden kann und in der eine Vielfalt an Lösungsvarianten durch (n) unabhängige Parameter (sogenannte „Bestimmungsgrößen“) erzeugt und eindeutig erfasst werden sollen. Jeder Parameter wird seinerseits durch eine diskrete Ausprägung (eine „Komponente“) spezifiziert. Durch die Kombination der Parameter soll laut Zwicky ein „totaler“ Überblick aller möglicher Lösungen eines vordefinierten Problems entstehen. Entsprechend dem Anspruch an Wiederholbarkeit, dem wissenschaftliche Methoden gemeinhin unterliegen, hat auch Zwicky für das genannte morphologische Verfahren fünf Operationalisierungsschritte fixiert: ${ }^{73}$

-1. Schritt: Genaue Umschreibung und zweckmässige Verallgemeinerung des Problems;

-2. Schritt: Bestimmung und Lokalisation aller die Lösung des Problems bestimmender Parameter;

- 3. Schritt: Aufstellung des morphologischen Schemas oder des morphologischen Kastens, aus dem alle Lösungen des gegebenen Problems vorurteilslos herausgeschält werden;

- 4. Schritt: Bewertung aller Lösungen auf Grund eines bestimmt gewählten Wertestandards;

- 5. Schritt: Wahl der optimalen Lösung und Weiterverfolgung derselben bis zur fertigen Konstruktion.

Ersichtlich wird bei näherer Betrachtung des Vorgehens indes bald, dass man sich je nach Anzahl der gewählten Parameter und Komponenten mit einer schieren Fülle von Daten konfrontiert sieht. Es obliegt nunmehr dem subjektiven Urteil der jeweiligen Anwender und ihren individuellen Erfahrungen, „optimale“ Lösungen aus der Datenfülle zu extrahieren. Dieser Aspekt der subjektiven Auswertung und Bewertung der Resultate findet jedoch in den Schriften zur Morphologie nur wenig Aufmerksamkeit. Ebenso wenig wird der Umstand thematisiert, dass bereits die 
Auswahl und Anordnung der Parameter den Lösungsraum entscheidend vordefinieren. Stattdessen wird zugunsten der Methode argumentiert, dass eine „morphologische Übersicht" über die „Totalität" von Phänomenen, Prozessen, Vernetzungen dem Problemlöser bereits dabei helfen könne, die Denkarbeit $\mathrm{zu}$ ordnen und $\mathrm{zu}$

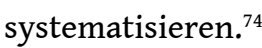

Doch scheint dieses rationalistische Argument für Zwicky mit Blick auf die Bedeutung seiner morphologischen Methoden nur zweitranging gewesen zu sein. Sehr viel stärker akzentuierte er die Notwendigkeit systematischer Problemlösungs- und Ideenfindungsmethoden vor der Kulisse drängender Gesellschaftsprobleme und drohender Zukunftsszenarien in der Kriegs- und Nachkriegszeit, ${ }^{75}$ namentlich im Hinblick auf einen möglichen atomaren Krieg. Die technisch-funktionale Dimension seiner Methoden wird hier um eine gesellschaftlich-moralische erweitert. Bezeichnend hielt er dazu fest: „Die Atomphysik hat uns nach dem Abwurf der Atombomben auf Hiroshima und Nagasaki vor die Wahl gestellt: Entweder Eine Welt oder Keine." ${ }^{\text {"76 }}$ Aus seiner Sicht konnte die Option auf den Fortbestand „einer Welt" nur mittels eines ganzheitlich-systematischen, also morphologischen Denkens verwirklicht werden. ${ }^{77}$ Mithin ist der von ihm propagierte Morphologe als ein „Spezialist des Unmöglichen“ $\mathrm{zu}$ verstehen. ${ }^{78}$ Morphologische Methoden schienen ihm unübertroffen geeignet, um auf vorurteilsfreie Weise über Fragen des Zivilschutzes, Probleme des Luft- oder Unterseekampfs, „die Möglichkeit von Atombomben“ oder strategische Kriegsführung ${ }^{79}$ nachzudenken. Für ihn war es mithin nur „logisch mit der [...] morphologischen Methode eine Analyse aller möglichen Arten von Kriegsführungen zu unternehmen“, und bereits Ende der 1930er Jahre stellte er den „morphologische[n] Ansatz für Verteidigungsprobleme" als einzig sicheren Weg in Aussicht, um der "Vernichtung" durch faschistische Diktaturen“ zu entgehen. ${ }^{80}$ Prägnant subsummierte er solche kriegstechnisch-methodischen Aspekte der Morphologie unter dem Stichwort einer „Morphology of War". ${ }^{81}$

Morphologisches Denken versprach, vergleichbar mit einer militärstrategischen Operation, systematisch Überblick und Ausblick erzeugen. Auch versprach es, ein umfassendes und objektives Wissen $\mathrm{zu}$ generieren, das aufgrund seiner wissenschaftlich-systematischen Grundlegung von allen Vorurteilen und Denkblockaden ,gereinigt‘ wäre: „Voraussetzung für das morphologische Vorgehen und für das Erzielen eines optimalen Erfolges ist absolute Vorurteilslosigkeit sowie umfassende Kenntnis der wesentlichste Stützpunkte menschlichen Wissens." ${ }^{\text {"2 }}$ Die Hoffnung, die Zwicky in seine Methoden setzte, wurde also wesentlich durch seinen Glauben an wissenschaftliches Wissen, und mehr noch, an eine vermeintlich überzeitlich gültige und wertefreie wissenschaftliche objektivität und Neutralität genährt. Sowohl die Ausgangspunkte als auch die Resultate morphologischer Analysen sollten durch eine wissenschaftliche Fundierung objektiviert und neutralisiert werden.

Zwicky versuchte auf diese Weise, sich über die oft ideologisch geführten Fortschrittsdebatten im Kalten Krieges hinwegzusetzen, auch versuchte er, den Wissenschaften nach den Greueltaten des Zweiten Weltkriegs wieder einen produktiven Platz bei der Gesellschaftsentwicklung zuzuordnen. Ohne die Wissenschaftler, so schreibt Zwicky, wären „Erzverbrecher wie Lenin, Stalin, Hitler und Mao-Tse-Tung nicht weit gekommen“, es sei deshalb „höchste Zeit, dass sie sich endlich selbstlos daran machen, an den grossen Problemen zum Aufbau einer vernünftigen, freien und gesunden Welt mitzuarbeiten“ ${ }^{.83}$ Dennoch war er dem Stereotyp des oben 
idealtypisch skizzierten antiautoritären kreativen Charakter gerade dort auch meisten verhaftet, wo er vorurteilsfreies Denken unhinterfragt als ein westliches Ideal propagierte ${ }^{84}$. Zugespitzt formuliert kann man schließen, dass paradoxerweise gerade das Postulat, unter dem Mantel von Wissenschaftlichkeit undogmatisch, offen und kreativ zu denken, selbst zum blinden Fleck und zum ideologisierten Signum vermeintlich westlicher Vorurteilslosigkeit während des Kalten Krieges avancierte.

\section{Kreativitätsforschung im Sozialismus}

Sowjetunion während der Nachkriegsjahre und des Kalten Krieges versucht, Kreativität systematisch für die nationalen wirtschaftlichen und politischen Interessen nutzbar zu machen. Auch dort galt Kreativität - in der Regel wurde von ,Schöpfertum` oder, in einem technischen Sinne, von ,Erfindertum' gesprochen- als eine nicht zu vernachlässigende wirtschaftliche Ressource und Produktionskraft. Kaum bekannt ist heute, dass nach dem Zweiten Weltkrieg auch in der Sowjetunion gezielte Bestrebungen stattfanden, das Erfindertum zu verwissenschaftlichen und so für jeden erlernbar zu machen. Diese Bestrebungen waren jedoch nicht unumstritten, sie wurden wechselnd von aktiver Förderung, toleranter Duldung oder aber Widerstand seitens der sozialistischen Regimes flankiert.

Was den Stand der Kreativitätsforschung in der Sowjetunion in der Nachkriegszeit betrifft, so ist sie mit jener in der USA nur schwer vergleichbar, da sich beide Diskurse von den politischen Systemen und Ideologien sowie den realen Arbeitsbedingungen ganz abgesehen - auf divergente theoretisch-psychologische Traditionen beziehen (die ihm Rahmen dieses Textes auch nur punktuell benannt werden können). Es gab zwischen den beiden Diskursen jedoch nicht nur Unterschiede, sondern auch Verbindungen: trotz des ,Eisernen Vorhangs' waren Kreativitätsforschende in der Sowjetunion über Entwicklungen in der westlichen Arbeits- und Kreativitätsforschung gut informiert. In den einschlägigen Texten lassen sich etliche Verweise auf westliche Autoren und deren Befunde finden. Umgekehrt trug die intensive Rezeption der USamerikanischen Kreativitätsforschung in der BRD um 1970 dazu bei, dass neben westlichen auch sozialistisch-marxistische Ansätze in die Diskussionen über Kreativität miteinbezogen wurden. Mechthild Curtis, von der einen solcher Versuch (mit Schwerpunkt auf psychoanalytische Kreativitätstheorien) stammt, konstatierte, dass die UdSSR-Forscher „reichhaltig europäische und amerikanische Beiträge aus verschiedensten Ländern“ zitierten und dass ihre „interdisziplinäre Kooperation“ ihnen „internationale Belesenheit und Informiertheit“ ermögliche ${ }^{85}$. Doch merkt sie ebenfalls an, dass dieses Interesse seitens sowjetischer Forscher eine „stark einseitige Transparenz" aufweise ${ }^{86} \mathrm{da}$ im anglophonen Raum nur wenig über die entsprechenden sozialistischen Ansätze bekannt sei.

Was nun die theoretisch-psychologische Basis sozialistischer Kreativitätsdebatten betrifft, so wurde einerseits, und naheliegender Weise, der Zusammenhang zwischen individueller und gesellschaftlicher Schöpferkraft materialistisch-dialektisch begründet. Im Marxismus-Leninismus sollte es „kein gesellschaftliches Schöpfertum ohne individuelles Denken und Handeln der Werktätigen und kein individuelles ohne gesellschaftliches Schöpfertum geben“ ${ }^{87}$ Der „Zusammenhang zwischen individuellem schöpferischem Denken und Handeln und gesellschaftlichem Schöpfertum“ wurde als 
ein "objektiver" postuliert, wobei vor allem aber der gesellschaftlich-ideologische Überbau für das Individuum als handlungsanleitend galt. „Das gesellschaftliche Schöpfertum“, so wurde argumentiert, gebe „nicht nur dem individuellen Denken und Handeln die allgemeine Richtung“, sondern sei zugleich „Voraussetzung, Bedingung und Resultat des massenhaften Schöpfertums der Werktätigen“".88

Andererseits scheint der Kongruenz von künstlerischen und wissenschaftlichen Schaffensprozessen in den sozialistischen Kreativitätsdiskursen in gewisser Weise eine stärkere Bedeutung zuzukommen als in den USA. Margarete Vöhringer weist in diesem Zusammenhang auf ein wesentliches Charakteristikum sowjetischer Wissensgeschichte hin, dass nämlich nicht „die Differenzen von Kunst und Wissenschaft oder ihre Gemeinsamkeiten [...] im postrevolutionären Russland relevant [waren], sondern das fast vollkommene Fehlen dieser akademischen Unterscheidung“, „Künstler und Wissenschaftler scheinen auf einem gemeinsamen Terrain agiert zu haben“, so Vöhringer. ${ }^{89}$ Basierend auf dieser Engführung von Kunst und Wissenschaft wurden in den 1920er und -30er Jahren künstlerisch-wissenschaftliche Institutionen und Laboratorien gegründet, wie die Moskauer VchUTEMAS (Höhere KünstlerischTechnische Werkstätten) oder das GINKhUK (Staatliches Institut für Künstlerische Kultur), in denen nebst bekannten Künstlern wie Kandinsky, Tatlin oder Malevich zahlreiche weitere russische Kunstschaffende und Wissenschaftler mitwirkten..$^{90}$ Ein gemeinsamer Gegenstand dieser künstlerisch-wissenschaftlichen Kooperationen bildeten die $\mathrm{zu}$ jener Zeit sich ausbreitenden Lebenswissenschaften Physiologie, Psychologie und Psychotechnik ${ }^{91}$ und damit einhergehend die Frage nach der wechselseitigen Durchdringung von psychologischer Theorie und künstlerischem Schaffen..$^{92}$ So versuchte etwa der Komponist, Maler und Theoretiker Mikhail Matiushin basierend auf einer Erweiterung der Sinneswahrnehmung, eine Farbtheorie für die Belange von Architektur und Design zu entwickeln. ${ }^{93}$

Doch nicht immer waren die Künste in der Exploration des schöpferischen Schaffensprozesses gleichermaßen präsent. Bisweilen agierten sie als nur als untergeordneter Bestandteil einer allgemeinen Theorie sozialistischen Schaffens - ein Schaffen, dass der Psychologe Sergej Rubinstein kennzeichnend als die „grundlegende, historisch ursprüngliche Form menschlicher Tätigkeit" charakterisierte. ${ }^{94}$ Bereits zwischen 1907 und 1923 erschienen von unterschiedlichen Autoren acht Bände in einer Reihe zu Fragen der Theorie und der Psychologie des Schaffens. ${ }^{95}$ Sie stellen nach Curtis womöglich den Beginn einer sozialistischen ,Kreativitätsforschung dar, ${ }^{96}$ wenngleich die Qualität und Ausrichtung der Bände auch angezweifelt wurde. Namentlich äußerte sich der sowjetische Literaturwissenschaftler Boris Meilach, der in den 1960er Jahren selbst an einer Psychologie des künstlerischen Schaffens arbeitete, ${ }^{97}$ despektierlich über dieses Werk. Seines Erachtens fehlten in den Bänden „völlig Arbeiten über die Methodologie und Methodik der Psychologie des Schaffens, ja, in der Definition dieser Disziplin und ihres Gegenstandes selbst herrscht keinerlei Klarheit“". ${ }^{98}$ Weiter beklagte er, dass „das Spezifikum des künstlerischen Schaffens“ ignoriert werde und „mit dem wissenschaftlichen, technischen, handwerklichen Schaffen" zusammenfalle. ${ }^{99}$

In der Nachkriegszeit stellten arbeitspsychologische Ansätze, wie sie etwa Rubinstein 1946 in seinen umfangreichen Grundlagen der allgemeinen Psychologie unter dem Kapitelpunkt Die Arbeit behandelte, weiterhin eine wesentliche theoretische Grundlage für sozialistische Kreativitätsdebatten dar. ${ }^{100}$ Die künstlerische Arbeit bildete dort neben der „Arbeit des Arbeiters“, der „Arbeit des Erfinders“ und der „Arbeit des 
Wissenschaftlers" nur eine Kategorie unter anderen. Das psychologische Verständnis von Arbeit bezog sich in sozialistischer Lesart, so auch bei Rubinstein, nicht allein auf die „die Schaffung eines bestimmten Produkts“, sondern galt als „das wichtigste Mittel zur Formung der Persönlichkeit" überhaupt. ${ }^{101}$ Überraschend ähnlich wie in der Nachkriegskreativitätsforschung der USA wurde auch in der Sowjetunion eine Koppelung individueller und kollektiver Schöpfungskräfte angestrebt, bei der die "gesellschaftlichen und persönlichen Interessen [zusammen] fallen“ sollten. ${ }^{102}$ Freilich wurde diese vordergründig verwandte Zielsetzung in der Sowjetunion mit anderen Mittel durchgesetzt als in den USA. Auch distanzierten sich die sozialistischen Autoren in der Regel von den Ideologien und Praktiken kapitalistischer Arbeitsorganisation - ja viel grundlegender noch wurde das westliche Interesse an Kreativität in der Nachkriegszeit als ein Mangelsymptom fehlgeleiteter kapitalistischer Arbeitsteilung in Hand- und Kopfarbeit gedeutet. In diesem Sinne konnte, oder vielmehr durfte, es in sozialistischen Ländern „keine Kreativitätsforschung im Sinne der amerikanischen“ geben. ${ }^{103}$ Bei Rubinstein findet sich entsprechend dieser Überzeugung die folgende Ausführung:

Die Trennung von körperlicher und geistiger Arbeit in der kapitalistischen Gesellschaft führt dazu, daß dort die Arbeit des Arbeiters je nach Mechanisierung der Produktion immer mechanisierter und geistloser wird. So erklärt Ford geradeheraus, daß die von ihm eingeführte Organisation der Produktion speziell auf die Senkung der Anforderungen gerichtet ist, die an die geistigen Fähigkeiten des Arbeiters gestellt werden. Im Sowjetstaat ist alles darauf angelegt, gerade diese Fähigkeiten zu entwickeln, indem man den Gegensatz zwischen körperlicher und geistiger Arbeit überwindet. ${ }^{104}$

Dieser Lesart, dass die kapitalistische Arbeitsteilung die individuellen Fähigkeiten des Arbeites beschneide, wurde seitens der sozialistischen Arbeitstheorie das Idealbild eines umfassend gebildeten Arbeiters gegenübergestellt, der Hand- und Kopfarbeit zu verbinden wusste. Ungeachtet aber der divergierenden Arbeitsideologien in sozialistischen und kapitalistischen Produktionssystemen, lassen sich dennoch auf der Ebene der konkreten Arbeitspraktiken wiederum auch Gemeinsamkeiten zwischen den Systemen ausmachen. Vergleichbar wie im US-amerikanischen Kreativitätsdiskurs wurden auch in der Sowjetunion nach Ende des Zweiten Weltkrieges und während des Kalten Krieges eine Reihe von Kreativitäts- und Ideenfindungstechniken entwickelte, vermittels derer das sozialistische Projekt, den Gegensatz zwischen körperlicher und geistiger Arbeit zu überwinden, konkretisiert werden sollte. Darüber hinaus waren auch in der Sowjetunion der angestrebte nationale Wirtschaftserfolg sowie die internationale technologische Wettbewerbsfähigkeit entscheidende Motivatoren für die Genese eines systematisch fundierten, massenhaft betriebenen ,Erfindertums' und für eine pragmatische „Dialektik des Entwerfens““. ${ }^{105}$

\section{Theorie zur Lösung erfinderischer Probleme}

Charakteristisch und zugleich herausragend im Kontext sozialistischer Kreativitätsförderung ist die bis heute in Management- und Ingenieurskreisen ${ }^{106}$ bekannte Theorie zur Lösung erfinderischer Probleme, Teoria reschenija isobretatjelskich sadatsch, kurz TRIS genannt. TRIS wurde nach dem Zweiten Weltkrieg wesentlich durch den russischen Ingenieur und Marineleutnant Genrich Soulovich Altschuller (1926-1998) entwickelt. ${ }^{107}$ Ähnlich wie schon im Zusammenhang mit Fritz Zwicky erwähnt, so wird auch Altschuller nachgesagt, sich bereits in seiner Jugendzeit intensiv 
mit dem Erfindertum beschäftigt zu haben. Im Jahre 1946 soll Altschuller, damals gerade 20 Jahre alt, mit einer seiner ersten Erfindungen - einer Methode um ohne Taucherausrüstung einem havarierten U-Boot zu entkommen - das Interesse des Militärs auf sich gezogen haben. ${ }^{108}$ Nach Abschluss der Militärakademie trat er eine Stelle beim Patentamt der Kaspischen Marine an. Während dieser Tätigkeit sichtete er gemeinsam mit seinem ehemaligen Studienkollegen Raphael Shapiro zehntausende von Patentschriften und beriet Erfinder bei ihren Projekten. Auf der Basis dieses umfangreichen empirischen Studiums versuchte er sodann, wissenschaftliche Gesetzmäßigkeiten und Regeln zu identifizieren, die zu erfolgreichen technischen Erfindungen geführt haben. Das intensive Studium bereits gestellter Aufgaben und getätigter Erfindungen bildeten den methodischen Grundstein in Altschullers Theorie. Er hielt diesbezüglich fest: „Man muß die Erfahrungen der Erfinder studieren und die charakteristischen Merkmale gute Lösungen herausarbeiten, durch die sie sich von schlechten Lösungen unterscheiden. Die Schlußfolgerungen können bei der Lösung von Erfindungsaufgaben verwendet werden. “" ${ }^{109}$

Die Anwendung von TRIS sollte das Denken so organisieren, „als ob einem die Erfahrungen aller oder zumindest sehr vieler talentierter Erfinder zu Verfügung stehen ". ${ }^{110}$ Patente boten sich als Grundlage für eine systematische Erfindungslehre insofern an, da sie als materialer Wissensspeicher in bereits "aufbereiteter Form“ vorlagen: „ausserordentlich umfangreich, alle möglichen Gebiete erfassend, klassifiziert, in exakter Darstellungsform und ständig ergänzt." "111 Aus der schieren Masse der von ihm analysierten Patente extrapolierte Altschuller rund 35-40 Prinzipen erfolgreichen Erfindens. ${ }^{112}$ Schenkt man den Angaben in der TRIS-Literatur Glauben, soll er zu seinen Lebzeiten einige zehntausend Patente gesichtet haben. Überhaupt gilt das Total der im Verlauf der TRIS-Entwicklung untersuchten und analysierten Patenschriften (mittlerweile sollen es 2.5 Millionen Patente sein) als Garant und Massstab für ihr methodisches Gelingen ${ }^{113}$ Altschuller selbst spricht davon, dass ein aussagekräftiges „Informationsmassiv“ an Patentschriften um die 20.000-30.0000 Patente mindestens umfassen solle. ${ }^{114}$

Mit TRIS wandte sich Altschuller gegen die weithin verbreitete Auffassung, dass der kreative Prozess nicht plan- oder steuerbar sei sowie auch gegen den Umstand, „dass Erfinder nicht gern davon [erzählen], wie sie zu neuen technischen Ideen kamen“. ${ }^{115}$ „Warum“, so fragte er, kann „nicht der, der lernen kann und will, auch das Erfinden lernen?" ${ }^{\prime 16}$ Der Vorstellung, dass der kreative Prozess in „Kultur, Wissenschaft, Technik und Kunst die plötzliche Erleuchtung " sei, ${ }^{117}$ stellte Altschuller eine andere „Technologie“ entgegen, „die zwar dieselben Produkte ergab, nämlich Erfindungen, aber mit einem anderen Produktionsprozeß, einem der lenkbar war, gut organisiert und effektiv". ${ }^{118}$ Die ihn antreibende Vision war es also, über Zufallsergebnisse und ineffizientes ,Herumpröbeln“ beim Erfindungsprozess hinauszukommen „mit dem Ziel, effektive Methoden für die Lösung von Erfindungsaufgaben zu schaffen“ ". ${ }^{119}$

41 Analog zu Fritz Zwicky, der zeitgleich in den USA mit seinen morphologischen Methoden an einer "Systematisierung der Erfindungsgabe" auf praktischer Ebene arbeitete, ${ }^{120}$ wollte auch Altschuller für den sowjetischen Erfinder „eine praktisch anwendbare Theorie zur Lösung von Erfindungsaufgaben“ schaffen und „die Grundprinzipien einer wissenscha[f]tlichen Organisierung der schöpferischen Erfindungstätigkeit" aufstellen. ${ }^{121}$ Sein Fokus lag dabei vor allem auf technischen Problemen, doch stand - zumindest spekulativ - auch ein „Lösungsalgorithmus für 
wissenschaftliche Probleme“ im Raum. ${ }^{122}$ Der Aspekt der Wissenschaftlichkeit ist ohnehin bezeichnend für Altschullers Zugang zum Erfindertum und spiegelt womöglich ein grundlegendes Interesse des sozialistischen Wissenschaftsdispositivs an nomologischen Theorien wider - sei es nun mit Blick auf die Natur oder die Gesellschaft. In einem seiner Buchtiteln ist denn auch bezeichnenderweise vom „Schöpfertum als exakte Wissenschaft" (1979) die Rede, auf englisch übersetzt erschien das Buch als „Creativity as an Exact Science“ (1984). ${ }^{123}$ Zurückhaltender hingegen mit dem Versprechen der Wissenschaftlichkeit war man bei einer deutschen Ausgabe in der DDR (1986), hier ist schlicht die Rede von „Wege[n] zur Lösung technischer Probleme“. 124

Altschuller stellte in seinen Büchern nicht nur theoretische Hypothesen zum Prozess des Erfindens vor, sondern gab den Erfindern gezielte praktische Anweisungen. Mit ARIS, einem „Algorithmus zur Lösung von Erfindungsaufgaben“ konzipierte er einen konkretes „Handlungsprogramm“, dessen Hauptmerkmale „die Determiniertheit, die Anwendungsbreite und die Wirksamkeit" waren. ${ }^{125}$ ARIS stellt einen, wenn nicht den zentralen methodischen Bestandteil der Theorie TRIS dar, bestehend aus einer Liste von über 80 Schritten, die zur erfolgreichen Erfindung führen sollen. In Konsequenz zu der Wissenschaftlichkeit die Altschuller für seinen Ansatz einforderte, sollten die Schritte von ARIS mittels konsequenter Anwendung und Überprüfung durch Peers stetig überprüft und optimiert werden, entsprechend tragen auch die verschiedenen ARIS-Formen die Jahreszahlen ihrer Überarbeitung: ARIS-56, ARIS-61, ARIS-68, ARIS-77, ARIS-85, ARIS-91...

Wiederum vergleichbar mit Zwicky, versuchte auch Altschuller, den komplexen Erfindungsprozess in diskrete, operationalisierte Schritte $\mathrm{zu}$ gliedern und ihn dergestalt in einem naturwissenschaftlichen Sinne repetierbar und intersubjektiv vermittelbar zu machen. „Bei Anwendung des Algorithmus nähert sich der Erfinder allmählich, Schritt für Schritt der Lösung“", so Altschuller. ${ }^{126}$ Einige Etappen dieses Weges seien „fast ganz nach den Gesetzen der Logik aufgebaut“, zuweilen trete die Logik aber etwas zurück und dann dränge ,der Algorithmus die Vorstellungskraft des Erfinders in die erforderliche Richtung“ und schaffe "die Bedingungen für das Entstehen einer Intuition " ${ }^{127}$ Analog dazu ist der Erfindungsalgorithmus ARIS in drei Stadien unterteilt: in ein analytisches, ein operatives und ein synthetisches. ${ }^{128}$ Diese Stadien sind ihrerseits durch konkrete Anweisungen und Fragen an den Erfinder gegliedert. So wird er beispielsweise im analytischen Stadium angewiesen: „Erster Schritt: Die Aufgabe ist in allgemeiner Form zu stellen. Zweiter Schritt: Man stelle sich das als ideales Endergebnis vor. Dritter Schritt: Was hindert an der Ausführung. Vierter Schritt: Wodurch? Fünfter Schritt: Unter welchen Bedingungen kann das Störende beseitigt werden?" ${ }^{129}$ Schritt für Schritt sollte man sich so einer erfolgreichen Erfindung annähern. 
Abb. 3. - Morphologischer Kasten

\begin{tabular}{|c|c|c|c|c|c|}
\hline \begin{tabular}{|l} 
PARAMETERS - \\
- WHAT THE SUBUEC \\
MUST 'BE' OR 'HAVE' \\
i.e. ITS REQUIRED \\
'CHARACTERISTICS'
\end{tabular} & $\begin{array}{c}\text { - TR } \\
\text { WHAT }\end{array}$ & $\begin{array}{l}\text { PARAME } \\
\text { HE ME, } \\
\text { DESCRII } \\
\text { THE } \\
\text { OR. }\end{array}$ & $\begin{array}{l}\text { ETER } \\
\text { ANS' OF } \\
\text { PTION } \\
\text { SUBJECT } \\
\text { HAVE. }\end{array}$ & $\begin{array}{l}\text { STEPS } \\
\text { ACHIEVII } \\
\text { OF - } \\
\text { MUST. }\end{array}$ & $e^{\frac{5}{5}}$ \\
\hline FORM & $\begin{array}{l}\text { CIRCULAR } \\
\text { CYLINDER }\end{array}$ & $\begin{array}{l}\text { RECTANGE. } \\
\text { CYLINDER }\end{array}$ & SPHERE & & \\
\hline SIZE & IUNIT & 2UNITS & 3UNITS & 4UNITS & $\begin{array}{l}\text { OEFINTION } \\
\text { OF UNNT } \\
\text { REQURED } \\
\end{array}$ \\
\hline MATERIAL & METAL & WOOD & PLASTIC & & \\
\hline ORIENTATION & $\underset{A X \mid S}{X}$ & $\begin{array}{c}Y \\
\text { AXIS }\end{array}$ & $\underset{\text { AXIS }}{Z}$ & & $\begin{array}{l}\text { DEFINITION } \\
\text { REQUIRED }\end{array}$ \\
\hline QUANTITY & 1 & 2 & 3 & 4 & \\
\hline SPEED & LOW & MEDIUM & HIGH & & $\begin{array}{l}\text { DEFINITION } \\
\text { REQUIRED }\end{array}$ \\
\hline ETC. & & & & & \\
\hline
\end{tabular}

Abb. 4. - Matrix der Widersprüche TRIS
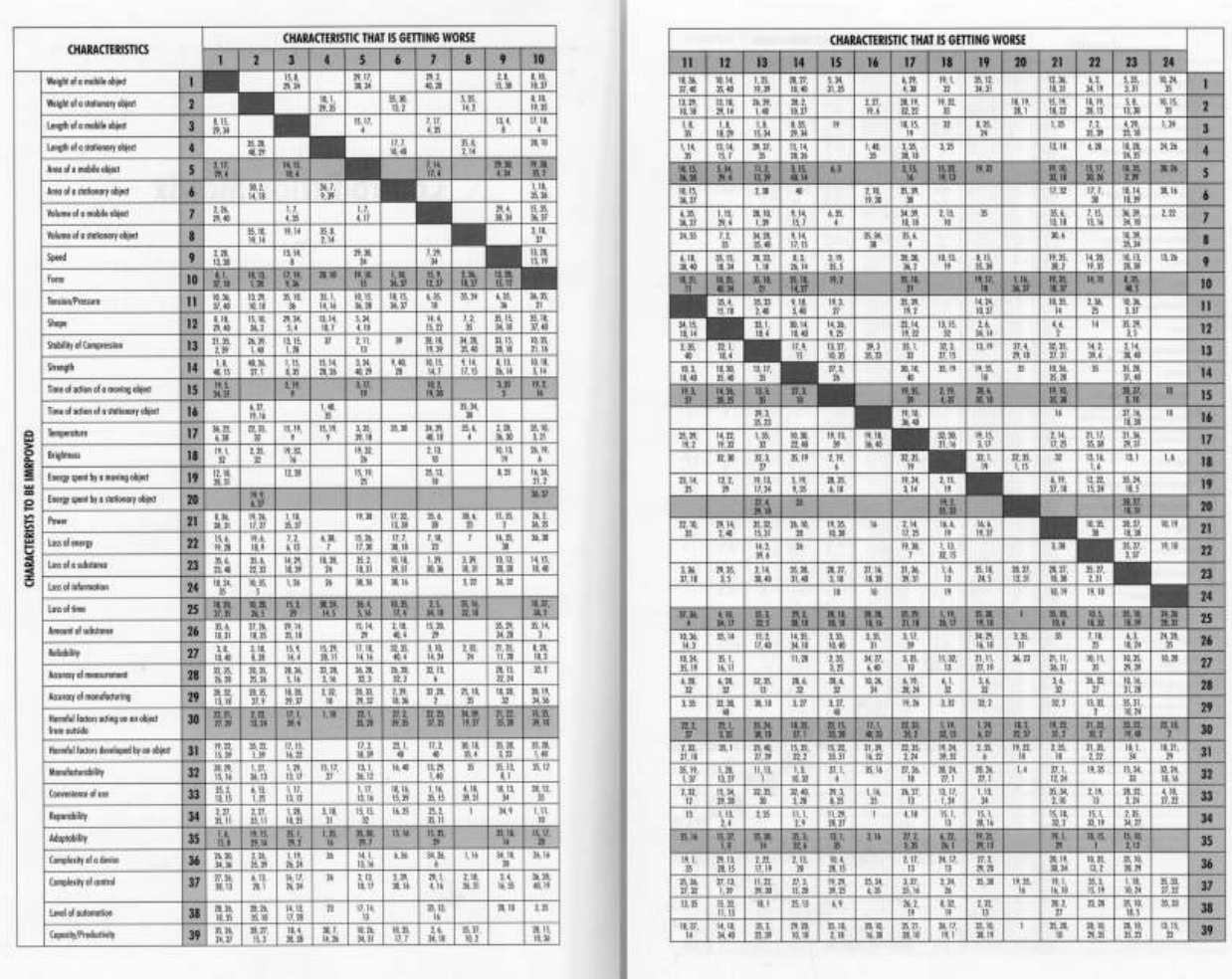

Rückt man nun die transnationale Dimension von Kreativitätstechniken in der Nachkriegszeit wieder ins Blickfeld, so ist von Interesse, dass Altschuller den USamerikanischen Kreativitätsdiskurs der Zeit gut kannte und sich in seinem Schriften 
konkret auf westliche Methoden wie Alex Osborns Brainstorming-Technik, Fritz Zwickys morphologische Analyse oder die Arbeiten der Synektik-Gruppe bezog. ${ }^{130}$ In seinen TRIS-Schriften versuchte er jedoch sich von diesen Methoden abzugrenzen, indem er ihnen ihre Eignung für die Lösung komplexer Erfindungsaufgaben absprach ${ }^{131}$ und für seine Theorie letztlich eine größere Wissenschaftlichkeit aufgrund ihrer empirisch abgeleiteten Systematik beanspruchte. Der prinzipielle Mangel der genannten westlichen Methoden bestehe darin, so Altschuller, dass sie für komplizierte Aufgaben nicht geeignet seien und deswegen in der Sowjetunion auch keine Anwendung fänden. ${ }^{132} \mathrm{Er}$ betonte zudem die Pionierrolle der Sowjetunion, da dort bereits „an der Erforschung der objektiven Gesetzesmäßigkeiten des Erfindens gearbeitet" wurde bevor der Begriff des „heuristischen Programmierens" als amerikanischer Terminus überhaupt auftauchte. ${ }^{133}$ Dessen ungeachtet liess er sich aber durchaus von westlichen Methoden inspirieren und nahm gewisse Aspekte daraus in seinen Theoriebaukasten auf. So findet sich etwa in der TRIS an prominenter Stelle mit der so genannten „Matrix der Widersprüche“ eine diagrammatische Konstruktion wieder, die deutlich an Zwickys morphologischen Kasten erinnert. ${ }^{134}$ [siehe Abb. 3 und 4]

Bezieht man nun die gesellschaftlich-politische Situation in der Sowjetunion in die Betrachtung von TRIS mit ein, so lässt sich das Bild einer höchst ambivalent rezipierten Theorie zeichnen. Auch war Altschuller aufgrund seiner Arbeiten grossen Gefahren und Repressionen unterworfen. Obwohl seine systematisierten Überlegungen zum Erfindertum zunächst auf großes Interesse stiessen, stagnierte das Projekt schon bald auf drastische Weise. 1948 beklagte Altschuller in einem Brief an Stalin die sowjetische Haltung gegenüber Innovation und Erfindung als "chaotisch und ignorant" und bot seine eigene Theorie zur Behebung dieses Missstands an. ${ }^{135}$ Sein Brief führte indes nicht zum erhofften landesweiten Durchbruch der TRIS, sondern erregte vielmehr Stalins Unmut. Altschuller wurde am 28. Juli $1950 \mathrm{zu} 25$ Jahren Arbeitslager wegen versuchter Sabotage verurteilt, er wurde jedoch bereits 1953, nach Stalins Tod, freigelassen und am 22. Oktober 1954 förmlich rehabilitiert. Die Zeit, die Altschuller in sibirischen Arbeitslagern verbringen musste, hat sich in der TRIS-Literatur zum narrativen Kern einer fast schon mythologisierten Ursprungserzählung der TRIS verdichtet. Es wird anhand unterschiedlichster Begebenheiten überliefert, dass Altschuller die desolaten Verhältnisse im Arbeitslager nur dank findiger Anwendungen seiner Theorie überleben konnte. ${ }^{136}$ Ungeachtet des kaum $\mathrm{zu}$ überprüfenden Wahrheitsgehalts dieser biographischen Erzählung so bleibt zu konstatieren, dass die TRIS durch dieses Narrativ ihren ursprünglichen Geltungsbereich, jenen des technischen Erfindens, massiv expandieren konnte und als eine existentielle Technik menschlichen Überlebens überhöht wurde. Die Strahlkraft dieses Narrativs reichte in Folge wiederum bis in die technischen Kontexte zurück und wird dort bis heute als immanenter Beleg für das Funktionieren der TRIS angeführt. Als vermutlich weitere Auswirkung des Narrativs wird der Theorie und ihren Methoden ein sehr grundlegendes gesellschaftliches Veränderungspotential zugeschrieben. TRIS verändere das Denken, schreiben überzeugte Anhänger noch heute - und mehr noch: „auch die ganze Zivilisation.“" ${ }^{137}$

Altschuller konnte nach seiner Rehabilitierung die Arbeit an TRIS zwar wieder aufnehmen, doch stiess er damit weiterhin neben Zustimmung auch auf Widerstand und Skepsis. In den Einleitungen zu seinen Büchern wird diese Ambivalenz fast durchgehend thematisiert, etwa wenn er schreibt: „Allein der Gedanke, daß die schöpferische Arbeit bestimmten Gesetzen gehorche [...] gilt vielerorts heute noch als 
Ketzerei. “" ${ }^{138}$ Die Idee, dass das Erfinden lehr- und lernbar sei, wurde kontrovers diskutiert. Namentlich konkurrenzierte das von Altschuller vertretene Ideal eines systematisch vorgehenden Erfinders solche Vorstellungen, in denen ein göttlicher Geistesblitz oder eine genialische Eingebung zum Erfindungserfolg führten.

Innerhalb des Theorie-Rahmens von TRIS wurde aber noch eine weitere Annahme zugleich mit besonderer Aufmerksamkeit und Skepsis bedacht. Es handelt sich dabei um den Aspekt des „Widerspruchs“. Altschuller deklarierte, dass eine Aufgabe „erst dann zu einer Erfindungsaufgabe“ werde, „wenn zu ihrer Lösung ein Widerspruch überwunden werden muß“. ${ }^{139}$ Die Widersprüche konnten sowohl technischer, physikalischer als auch organisatorischer Art sein. Mithin sollte auch der Prozess des systematischen Erfindens "widerspruchslösend“ wirken. ${ }^{140}$ Diese Forderung stiess namentlich in Ingenieurkreisen auf Skepsis, da die Vorstellung von inhärenten technischen Widersprüchen nur schwer mit dem Idealbild einer kontrollierten Technikentwicklung in Übereinstimmung gebracht werden konnte. ${ }^{141}$ Überdies ist $\mathrm{zu}$ vermuten, dass mit dem Aspekt des Widerspruches fast zwingend auch ein grundlegend revolutionäres Potential im schöpferischen Denken assoziiert wurde, dessen Aktivierung kaum im Interesse des Sowjetregimes gewesen sein dürfte. In dieser Hinsicht scheint der systemstabilisierende Effekt von Kreativität in der Sowjetunion stärker als in den USA im Vordergrund gestanden zu haben und viele Kontroversen, die rund um den Aspekt der Widerspruchslösung bei Erfindungen geführt wurden, hatten genau diesen Effekt im Auge.

Dennoch war - und das ist bildhaft gesprochen die Kehrseite der Medaille - die wirtschaftliche und gesellschaftliche Notwendigkeit, technische Erfindungen und Optimierungen quasi am Laufband produzieren zu können, auch in der Sowjetunion während der Nachkriegszeit und des Kalten Krieges omnipräsent. In einem Text über Dialektisches Denken und Schöpfertum von 1984 sprach sich etwa Hans-Georg Mehlhorn entschieden für die Systematisierung des Erfindungsprozesses in der Tradition von Altschuller aus. Mehlhorn benennt vorrangig zwei Aspekte:

Die Geschwindigkeit des wissenschaftlich-technischen Fortschritts unter den Bedingungen der sich ständig verschärfenden Systemauseinandersetzungen erfordert ein Umdenken in zweifacher Richtung. Notwendig ist eine hohe Kontinuität in der Erfindertätigkeit. [...] Der andere Aspekt betrifft die schöpferische Aktivität [...]. Das Aufspüren von Verbesserungsmöglichkeiten ist Angelegenheit jedes Beschäftigten, nicht nur des Forschungs- und Entwicklungskaders. ${ }^{142}$

Relevant an dieser Aussage ist, dass sich die Argumente westlicher und sozialistischer Kreativitätspragmatiker in dieser Hinsicht frappierend ähneln. Von beiden Seiten wurden radikale Veränderungen in einer hochtechnisierten Gesellschaft angeführt, um einen veränderten Umgang mit Kreativität zu begründen. Das Interesse beider Seiten galt zudem der systematischen Aktivierung einer kollektiven kreativen Schaffenskraft zugunsten der nationalen Produktionssteigerung. Die individuelle kreative Selbstfindung des Einzelnen sollte somit passgenau den einerseits kapitalistischfordistischen, andererseits den sozialistischen Bedürfnissen der Nachkriegsgesellschaften als Ganzes entsprechen. So wie seitens der US-amerikanischen Kreativitätspsychologie "von fast allen Menschen kreative Akte“ erwartet wurden, ${ }^{143}$ so wurde auch in der Sowjetunion Kreativität in bevorzugter Weise als ein Massenphänomen gesehen. Der Psychologe Rubinstein bekräftigte diese Wunschvorstellung mit den Worten: „Wir wissen jetzt aus Erfahrung, dass das Erfindertum eine Massenerscheinung ist. Es 
erwächst häufig aus der Arbeitspraxis und ist in der UdSSR unter den Werktätigen weit verbreitet.“" ${ }^{144}$

Rubinstein lag mit seiner Diagnose insofern nicht falsch, da sich die TRIS im Verlauf der Jahrzehnte ungeachtet ihrer Kritiker auf einer breiteren institutionellen Basis als „System gesellschaftlicher Erfinderschulen “ ${ }^{\text {" }} 45$ etablieren konnte. Nach Aussage von Altschuller gab es im Jahre 1978 „, bereits etwa 100 dieser Schulen (in Moskau, Leningrad, Baku, Wolgograd, Gorki und anderen Städten). Die Ausarbeitung der Theorie, der Erprobung und Vervollkommnung von ARIS sowie die Organisation der Ausbildung waren eine kollektive Arbeit, an der sich eine große Gruppe von Forschern beteiligte" ${ }^{146}$ Die Vermittlung von TRIS wurde systematisch betrieben, die Organisatoren waren „verschiedene Ministerien und Ämter, Leitungen von Betrieben, Forschungsinstituten und Hochschulen, Gebietskomitees und Stadtkomitees des Komsomol, die Unionsgesellschaft ,Znanie`, die Wissenschaftlich-Technischen Gesellschaften (NTO) sowie die Unionsgesellschaft der Erfinder und Rationalisatoren (VOIR) “. ${ }^{147}$ Konzipiert wurden Einführungsveranstaltungen im Umfang von 20 Stunden, darüber hinaus gab es eine „Schule des erfinderischen Schöpfertums“ mit 100-120 Lehrstunden sowie ein "Institut für erfinderisches Schöpfertum“, an dem die Ausbildung von TRIS-Fachleuten und Dozenten rund 220240 Stunden beanspruchte. ${ }^{148}$ Abgesehen von diesen TRIS-spezifischen Institutionen floss die Lehre aber auch in den allgemeinen technischen Unterricht in der Sowjetunion ein. In der Ukraine beispielsweise wurde in den 1980er Jahren das Fach „Grundlagen des technischen Schöpfertums“, basierend auf der Altschuller-Schule, als obligatorischer Unterrichtsbestandteil an den Technischen Hochschulen eingeführt. ${ }^{149}$

\section{Neuererbewegung in der DDR}

51 Auch in der DDR wollte man in dieser Hinsicht „von der Sowjetunion lernen“ und so gab es ab den 1970er Jahren ein reges Interesse an der Erfindungslehre Altschullers. ${ }^{150}$ 1973 erschien eine deutsche Ausgabe seines Buches Algoritm izobretenjia (1969) beim Tribüne Verlag Berlin unter dem Titel Erfinden - $(k)$ ein Problem. ${ }^{151}$ Weitere Übersetzungen folgten, so dass die TRIS auch im deutschen Sprachraum Fuss fassen konnte. ${ }^{152}$ Anfang der 1970er Jahre entwickelte sich in der DDR eine Reihe von Erfinderschulen, in denen ausgehend von kreativitätsfördernden Methoden wie TRIS bzw. ARIS, Brainstorming, Synektik und Morphologie „eine eigenständige, auf den [...] Bedarf zugeschnittene Erfindungsmethodik" entwickelt wurde. ${ }^{153}$ Diese Erfinderschulen wurden, nach Aussage ihrer Initiatoren, nicht auf „staatliche Weisung“154 sondern durch engagierte „Fach- und Führungskräfte ${ }^{\text {“155 }}$ initiiert, auch durchliefen das Erfindertum innerhalb der DDR im Verlauf der Jahrzehnte wechselnde, ambivalente Phasen der Anerkennung, aber auch der Behinderung und Retardierung. ${ }^{156}$ Die Erfindungssystematiker Hans-Jochen Rindfleisch und Rainer Thiel erinnerten sich 1994: „Kreative Menschen konnten in der komplizierten Realität der DDR Spielräume [...] entdecken. So wurden auch Spielräume für das Phänomen ,Erfinderschule` aufgedeckt und erschlossen. Am Ende, als schon alles zu spät war, gab es dann sogar allerhöchste Weisungen, die Erfinderschulen ernst zu nehmen. ${ }^{\text {"157 }}$

Eine Grundlage für die, wenn auch phasenweise gehinderte, Verbreitung des professionellen Erfindertums in der DDR war, dass es dort bereits Anfang der 1950er Jahre Bestrebungen zur Förderung eines massenhaften, das heisst auf der Arbeiterschicht basierenden Erfindertums gab. Nach sowjetischem Vorbild wurde eine 
Rationalisatoren- und Neuererbewegung (von russisch: nowator) ins Leben gerufen, „die sich um das kreative Potential der Werktätigen der DDR“ sorgen sollte. ${ }^{158}$ Der Staatsratsvorsitzende Walter Ulbricht erinnert sich 1966 in seinem Bericht zur Geschichte der Deutschen Arbeiterbewegung, dass die Neuererbewegung „bei der Erfüllung des ersten Fünfjahrplans“ eine wichtige Rolle gespielt hatte: „Das Neue des Plans der Rationalisatoren und Erfinder" bestehe darin, so führt Ulbricht aus, „daß die Bemühungen der Arbeiter, Angestellten, Techniker und Ingenieure, die Betriebsausrüstungen und den Produktionsablauf zu verbessern und die vorhandene Technik rationell auszunutzen, planmäßig zusammengefaßt und gefördert wurde“". ${ }^{159}$ Gemeinhin galten solche Verbesserungsvorschläge „als das Patent des kleinen Erfinders" ${ }^{160}$ da sich jeder Werktätige „im sozialistischen Wettbewerb" durch einen Erfindungs- oder Verbesserungsvorschlag auszeichnen konnte. ${ }^{161}$

Angedeutet ist in der zitierten Aussage von Ulbricht aber auch, dass sich die "Massenerscheinung“ des Erfindens - entgegen anders lautender Berichte (oder vielmehr Legenden $)^{162}$ - nicht einfach spontan aus „der Arbeitspraxis [...] der Werktätigen" heraus entwickelte, ${ }^{163}$ sondern Bestandteil staatlicher Planung war, die ihrerseits eines strengen Kontroll- und Wettbewerbssystems bedurfte. So war für Ulbricht der wichtigste Teil dieser Planung „die öffentliche Betriebsüberprüfung“, die vorsah, „daß die Brigaden gegenseitig ihren Arbeitsplatz, ihre Aggregate und deren technischen Zustand, die Arbeitsorganisation und den Materialverbrauch überprüfen" mit dem Ziel, „die Technik zu verbessern, die Arbeitsproduktivität zu steigern und die Selbstkosten zu senken“ ${ }^{164}$ Arbeiter und Angestellte wurden entsprechend „ständig angehalten, Verbesserungsvorschläge $\mathrm{zu}$ machen“, bei denen es um die „Rationalisierung von Produktionsabläufen, um Verbesserungen an vorhandenen Maschinen oder um bessere Produktionsbedingungen im Allgemeinen" ging. ${ }^{165}$

Dass aber genau diese „Produktionsbedingungen im Allgemeinen“ in der DDR tatsächlich Gegenstand offener „Verbesserungsvorschläge“ sein konnten, wurde seitens der BRD stark angezweifelt. In dieser Hinsicht wurde auch die Neuererbewegung in der DDR von westdeutscher Seite als eine Täuschung und Instrumentalisierung der arbeitenden Menschen kritisiert. In einer 1953 vom Bundesministerium für Gesamtdeutsche Fragen herausgegebenen Broschüre mit dem Titel Aus der Fabrik des Sowjetmenschen wird scharf kommentiert, dass „all diese Aktivisten, Brigadiers, Neuerer und Rationalisatoren“ nichts anderes seien "als Modelle, die in der Mehrzahl aus der Sowjetunion importiert wurden und nach denen nun auch der deutsche arbeitende Mensch geformt werden soll“" ${ }^{166}$ Die wirtschaftlichen Erfolge, die das DDR-Regime mit Blick auf die Neuererbewegung für sich proklamierte, wurden in dieser gegenstrebigen westlichen Lesart als ein sinnloses Anwachsen des Bürokratieapparates bei gleichzeitig zunehmender Ausbeutung der Arbeiternehmenden angeklagt:

Bezahlt wird dies alles dank der ,Neuerer'-Wirtschaft vom Arbeiter: durch weniger Lohn für mehr Arbeit, durch physische Überbeanspruchung und durch Phantasiepreise in den Staatsläden, aus deren Gewinnen das Investitionsprogramm für heruntergewirtschaftete Betriebe mitgespeist wird [...]. ${ }^{167}$

Sehr viel grundsätzlicher aber wurde seitens der BRD die psychologisch-ideologische Dimension sozialistischer Arbeitsorganisation kritisiert. Die sozialistische Definition von Arbeit als "das wichtigste Mittel zur Formung der Persönlichkeit" ${ }^{\text {“168 }}$ wurde in westlicher Lesart als ein "Instrument der Menschenformung“ in der diktatorisch verwalteten „Fabrik des Sowjetmenschen“ gesehen: „Der Mensch des Stalinismus soll ohne Rest in 
seiner Arbeit aufgehen; sein Wert erschöpft sich darin, daß seine Arbeitskraft als Wirtschaftsfaktor eingesetzt, verbraucht und ersetzt werden kann“, so heißt es in der besagten Broschüre aus der BDR. ${ }^{169}$

Konfrontiert man diese Aussage mit der fast zeitgleich geäußerten Absicht USamerikanischer Psychologen, mittels staatlich geförderter Kreativitätsforschung „eine Grundlage für die Mittel zur Auswahl von Menschen mit kreativem Potential ${ }^{{ }^{170}} \mathrm{zu}$ generieren, so drängt sich erstens die Frage auf, inwiefern diese Kritik nicht auch, zumindest im Ansatz, auf Arbeits- und Kreativitätskonzeptionen westlichkapitalistischer Produktionssysteme zurückgespiegelt werden müsste, um das signifikante Aufkommen von Kreativitätsdiskursen und -techniken in der Nachkriegszeit in einer transnationalen Perspektive besser verstehen zu können. Ebenso dringend schließt sich daran aber zweitens auch die Frage an, inwiefern es in, oder neben, einer staatlich gelenkten, ideologisierten Kreativitätsförderung für Einzelne individuell und punktuell möglich war, sich kreative Spiel- und Freiräume in ökonomischen, vor allem aber auch persönlichen Belangen zu erschließen.

Vorläufig zu resümieren wäre im Hinblick auf die erste Frage, dass das Phänomen der ,Kreativität ‘ und die Entwicklung von systematisch kreativitätsfördernden Techniken während der Nachkriegszeit und des Kalten Krieges sowohl in kapitalistischen als auch sozialistischen Ländern einen wichtigen Bestandteil nationalökonomischer und damit auch politischer Interessen darstellte. Ungeachtet der zweifellos existenten und keinesfalls zu vernachlässigenden Unterschiede in den jeweiligen Wirtschaftssystemen, Staatsideologien und vor allem in den jeweiligen Arbeits- und Lebensrealitäten der Menschen in Ost und West lassen sich doch mindesten auch vier Analogien in den zeithistorischen Debatten um Kreativität ausmachen.

Erstens wurde Kreativität in beiden Systemen in Abhängigkeit eines bestimmten Produktionsverhältnisses von schöpferischem Individuum $\mathrm{zu}$ Gesellschaft und Staat verstanden und auf der Basis eines für diese Zwecke geeigneten anthropologischen Schaffensmodells definiert. Zweitens wurde die nationale und internationale wirtschaftliche Wettbewerbsfähigkeit beiderseits als zentrales Argument angeführt, um Kreativität systematisch zu erforschen und massenhaft zu fördern. Drittens scheint Kreativität nur innerhalb ihrer Grenzen als geordnete und systemstabilisierende, nicht jedoch als revolutionäre und chaotische Kraft gebilligt und entsprechend gesteuert worden $\mathrm{zu}$ sein. Viertens schliesslich wurde in beiden Systemen der Topos der Wissenschaftlichkeit herangezogen, um sich damit von konkurrierenden Ansätzen in der Kreativitätsförderung und somit in letzter Konsequenz von konkurrierenden Gesellschafts- und Arbeitsideologien abzugrenzen.

Was die zweite Frage, nach den individuellen kreativen Spiel- und Freiräumen betrifft, so ruft uns Fabian Heubel in Erinnerung, dass der Begriff der Kreativität zwar wie kaum ein anderer „zur Verklärung des avancierten Kapitalismus“ - in unserem Fall auch des Sozialismus - tauge, dass er deswegen aber nicht einfach nur als eine "Ideologie“ verdammt werden könne. ${ }^{171}$ Vielmehr weist seine Lesart eines „Dispositivs der Kreativität“ zum einen auf die konkrete Materialität und die konkreten Alltagspraktiken der Kreativitätsförderung auch während der Nachkriegszeit und des Kalten Krieges hin, sie offenbart zum anderen aber auch den ambivalenten Charakter dieses Dispositivs, das sich zwar durch spezifische „Technologien der Macht“ konstituiert und stabilisiert, dennoch aber die Möglichkeit „einer Emanzipation von Kreativität“ potentiell offen lässt. ${ }^{172}$ 


\section{BIBLIOGRAPHIE}

ADORNo Theodor W., Dissonanzen. Musik in der verwalteten Welt, Göttingen, 1956.

ADORNo Theodor W. et al., The Authoritarian Personality, 2 Bände, New York, 1964.

Aligica Paul Dragos und WeInSTEIN Kenneth R., „Introduction“, in dies. (Hg.), The Essential Herman

Kahn: In Defense of Thinking, Plymouth, 2009, S. 1-5.

AltSCHULleR Genrich S., Erfinden (k)ein Problem? Eine Anleitung für Neuerer und Erfinder, Berlin, 1973.

-, Creativity as an Exact Science. The theory of the solution of inventive problems, übersetzt von Anthony Williams, New York et al., 1984.

—, Erfinden. Wege zur Lösung technischer Probleme, Berlin, 1986.

-, Erfinden. Wege zur Lösung technischer Probleme, limitierter Nachdruck der 2. Auflage. Cottbus, 1998.

AltSCHUlLER Genrich S. und SeLJUZKI A., Flügel für Ikarus. Über die moderne Technik des Erfindens, Moskau-Leipzig et al., 1983.

ANDERSON Harold H. (Hg.), Creativity and its Cultivation, New York, 1959.

BADIA Lola und BONNER Anthony, Ramon Llull: vida, pensament i obra literària, Barcelona, 1988.

BENDIXEN Peter, Kreativität und Unternehmensorganisation, Köln, 1976.

BONNER Anthony (Hg.), Doctor Illuminatus: A Ramon Llull Reader, Princeton, 1999.

Boos Evelyn, Das große Buch der Kreativitätstechniken, München, 2010.

BOURKE Joanna, Fear. A Cultural History, London, 2005.

BRANDSTETTER Thomas, PiAS Claus und VeHLKen Sebastian, „Think- Tank-Denken. Zur

Epistemologie der Beratung“, in dies. (Hg.), Think Tanks. Die Beratung der Gesellschaft, Zürich, Berlin, 2010, S. 17-56.

BRÖCKLING Ulrich, „Über Kreativität. Ein Brainstorming“, in Ch. Menke und J. Rebentisch (Hg.), Kreation und Depression. Freiheit im gegenwärtigen Kapitalismus, Berlin, 2011, S. 89-97.

COHEN-Cole Jamie, „The Creative American: Cold War Salons, Social Science, and the Cure for Modern Societ“", Isis, Vol. 100, Nr. 2, 2009, S. 219-262.

Comrey Andrew L., „Joy Paul Guilford 1897-1987. A Biographical Memoir", in National Academy of Sciences, Washington, 1993, S. 199-222.

CRAWFORD Robert P., The Techniques of Creative Thinking, Englewood Cliffs, 1954

CURTIS Mechthild, „Theoria in nuce“, in dies. (Hg.), Seminar. Theorien der künstlerischen

Produktivität, Frankfurt am Main, 1976, S. 9-61.

DER SPIEGEL, 3. April 1967, S. 123-140.

DER SPIEGEL, 30. September 1974, S. 175-177.

DRIESEN Oliver, „Die Meta-Erfindung“, Brand Eins, Nr. 3, 2008, S. 118-121.

Foucault Michel, „Technologien des Selbst“, in ders., L. H. Martin, H. Gutmann und P. Hutton (Hg.), Technologien des Selbst, Frankfurt am Main, 1993, S. 24-62. 
FUNKE Joachim, „Kreativitätstechniken“, in V. Nünning (Hg.), Schlüsselkompetenzen: Qualifikationen für Studium und Beruf, Stuttgart, 2008, S. 207-219.

GetZels Jacob W., Jackson Phillip W., Creativity and Intelligence, London-New York, 1962. GoETHE Johann Wolfgang, „Zur Morphologie. Erster Band“, in ders., H. J. Becker, G. H. Müller, J. Neubauer et al. (Hg.), Zur Naturwissenschaft überhaupt, besonders zur Morphologie, München, 1989, S. 9-269.

Gould Steven Jay, Der falsch vermessene Mensch, Frankfurt am Main, 1988.

GREINER Bernd, „Angst im Kalten Krieg. Bilanz und Ausblick“, in B. Greiner, C. T. Müller und D. Walter (Hg.), Angst im Kalten Krieg, Hamburg, 2009, S. 7-31.

GRUBER Howard E. et al. (Hg.), Contemporary Approaches to Creative Thinking, New York, 1964.

GuILFORD Joy Paul, „Creativity“, American Psychologist, Bd. 5, Nr. 9, 1950, S. 444-454.

-, ,Kreativität“, in G. Ulmann (Hg.), Kreativitätsforschung, Köln, 1973, S. 25-43.

HAGNER Michael und HÖRL Erich (Hg.), Die Transformation des Humanen. Beiträge zur Kulturgeschichte der Kybernetik, Frankfurt am Main, 2008.

Heubel Fabian, Das Dispositiv der Kreativität, Dissertation, Technische Universität Darmstadt, Darmstadt, 2000.

HoRN Eva, „War Games: Der Kalte Krieg als Gedankenexperiment“, in T. Macho und A. Wunschel (Hg.), Science \& Fiction. Über Gedankenexperimente in Wissenschaft, Philosophie und Literatur, Frankfurt am Main, 2004, S. 310-328.

Hounshell David, „The Cold War. RAND, and the Generation of Knowledge, 1946-1962“, Historical Studies in the Physical and Biological Sciences, Vol. 27, Nr. 2, 1997, S. 237-267.

HÜRLIMANN Werner, Methodenkatalog. Ein systematisches Inventar von über 3000 Problemlösungsmethoden, Schriftenreihe der Fritz-Zwicky-Stiftung, Nr. 2, Bern, 1981.

JANTSCH Erich, Technological Forecasting in Perspective, OECD, Paris, 1967.

JENNINGS Helen H., „Military Use of Sociometric and Situation Tests in Great Britain, France, Germany and the United States“, Sociometry, Vol. 12, Nr. 1/3, 1949, S. 191-201.

LERNER Leonid, „About the Author, Genrich Altschuller“, in G. S. Altschuller, The Innovation Algorithm: TRIZ, Systematic innovation and technical creativity, übersetzt von Lev Shulyak und Steven Rodman, Worcester, 2007.

LESLIE Stuart, The Cold War and American Science: The Military-Industrial-Academic Complex at MIT and Stanford, New York, 1993.

KAUfMANN Arnold et al., Moderne Methoden der Kreativität, München, 1972.

KolTZE Karl, SouchKov Valeri, Systematische Innovation. TRIZ-Anwendung in der Produkt- und Prozessentwicklung, München-Wien, 2011.

MAREIS Claudia, „Methodische Imagination. Kreativitätstechniken, Geschichte und künstlerische Forschung“", in M. Tröndle und J. Warmers (Hg.), Kunstforschung als ästhetische Wissenschaft. Beiträge zur transdisziplinären Hybridisierung von Wissenschaft und Kunst, Bielefeld, 2011a, S. 203-241. -, „Systematisierte Innovationen. Entdecken und Erfinden in Fritz Zwickys Morphologischem Weltbild“, in H. Müller (Hg.), Wissenskulturen. Bedingungen wissenschaftlicher Innovation, studien des Aachener Kompetenzzentrums für Wissenschaftsgeschichte, Bd. 12., Aachen (im Druck), 2011b. 
MARR Rainer, Innovation und Kreativität, Wiesbaden, 1973.

MATUSSEK Paul, Kreativität als Chance. Der schöpferische Mensch in psychoanalytischer Sicht, München, 1974.

MEHLHORN Hans-Georg, „Dialektisches Denken und Schöpfertum“, Deutsche Zeitschrift für Philosophie, Nr. 32/2, 1984, S. 53-162.

MEILACH Boris, „Die Psychologie des künstlerischen Schaffens. Einige Worte über die Literaturwissenschaft in der Reihe der anderen Wissenschaften“, in M. Curtis (Hg.), Seminar. Theorien der künstlerischen Produktivität, Frankfurt am Main, 1976, S. 251-279.

MORGAN John S., Improving Your Creativity on the Job, Vermont, 1968.

MÜLLER Roland, Fritz Zwicky. Leben und Werk des grossen Schweizer Astrophysikers, Raketenforschers und Morphologen, Glarus, 1986.

ORLOFF Michael A., Grundlagen der klassischen TRIZ. Ein praktisches Lehrbuch des erfinderischen Denkens für Ingenieure, Berlin et al., 2002.

Osborn Alex, Your Creative Power, New York, 1961.

PIAS Claus, „One-Man Think Tank: Herman Kahn, oder wie man das Undenkbare denkt“, in Zeitschrift für Ideengeschichte, Heft 3, 2009, S. 5-16.

PICKERING Andrew, The Cybernetic Brain: Sketches of another future, Chicago-London, 2010.

PREISER Siegfried, Kreativitätsforschung, Darmstadt, 1976.

RINDFLEISCH Hans-Jochen und THIEL Rainer, Erfinderschulen in der DDR. Eine Initiative zur Erschließung und Nutzung von technisch-ökonomischen Kreativitätspotentialen in der Industrieforschung - Rückblick und Ausblick, Berlin, 1994.

RoBIN Ron, „Wie das Denken in die Fabrik kam. Kampf um den Korridor im Kalten Krieg“, in Zeitschrift für Ideengeschichte, Heft 3, 2009, S. 17-32.

RowEN Henry S., „How we worked“, in R. Zarate und H. Sokolski (Hg.), Nuclear Heuristics. Selected Writings of Albert and Roberta Wohlstetter, Carlisle, 2009.

RUBINSTEIN Sergej L., Grundlagen der allgemeinen Psychologie, 5. durchgesehene Auflage, Berlin, 1962. SAMELSON Franz, „World War I Intelligence Testing and the Development Of Psychology“, Journal of the History of the Behavioral Sciences, 13, 1977, S. 274-282.

-, „Authoritarianism from Berlin to Berkeley: On Social Psychology and History“, Journal of Social Issues, Vol. 42, Nr. 1, 1986, S. 191-208.

SCHIFFLER Horst, Fragen zur Kreativität, Ravensburg, 1973.

SCHLICKSUPP Helmut, Innovation, Kreativität \& Ideenfindung, 2. Auflage, Würzburg, 1987.

ScHulz Christiane, „Der künstlerische Schaffensprozess. Zu den Arbeiten des Leningrader Literaturwissenschaftlers Boris Mejlach“, in M. Curtis (Hg.), Seminar. Theorien der künstlerischen Produktivität, Frankfurt am Main, 1976, S. 287-301.

SPECHT Robert Dickerson, „RAND: A Personal View of Its History“, Operations Research, Vol. 8, Nr. 6, 1960, S. 825-839, auf: <http://www.rand.org/pubs/papers/P1601>.

STEIN Stephan, Aus der Fabrik des Sowjetmenschen: Aktivisten, Neuerer, Rationalisatoren im Dienste der Ausbeutung der menschlichen Arbeitskraft, Presse- und Informationsstelle, Bundesministerium für Gesamtdeutsche Fragen, 1953, S. 20-24. 
STöcKLI Alfred, „Morphologie nach Zwicky“, in ders. und R. Müller, Fritz Zwicky. Astrophysiker. Genie mit Ecken und Kanten, Zürich, 2008, S. 226-242.

STÖCKLI Alfred und MÜLLER Roland, Fritz Zwicky. Astrophysiker. Genie mit Ecken und Kanten, Zürich, 2008, S. 226-242.

STRAUMANN Norbert und TAMMANN Gustav A., „Zwickys bedeutendste Beiträge zur Astrophysik und zur beobachtenden Astronomie", in A. Stöckli und R. Müller, Fritz Zwicky. Astrophysiker. Genie mit Ecken und Kanten, Zürich, 2008, S. 206-225.

STREBEL Heinz (Hg.), Innovations- und Technologiemanagement, Wien, 2007.

THIEL Rainer, „Einleitung. Die Aktualität eines unbequemen Werkes“, in G. Altschuller, Erfinden. Wege zur Lösung technischer Probleme, limitierter Nachdruck der 2. Auflage, Cottbus, 1998, S. 13-15. Tillberg Margareta, Coloured Universe and the Russian Avant-Garde. Matiushin on Colour Vision in Stalin's Russia, 1932, Stockholm, 2003.

ULBRICHT Walter, Geschichte der deutschen Arbeiterbewegung, Hg. zum Parteilehrjahr 1966/67 auf Beschluss des Politbüros des ZK der SED vom 5. Juli 1966, Berlin, Bd. 13, 1969, S. 170-248, auf: <http://www.ml-werke.de/andere/ul13_186.htm>, PDF S. 1-11.

UlmanN Gisela, „Einleitung. Psychologische Kreativitätsforschung“, in dies. (Hg.):

Kreativitätsforschung, Köln, 1973, S. 11-22.

VöHRINGER Margarete, Avantgarde und Psychotechnik: Wissenschaft, Kunst und Technik der Wahrnehmungsexperimente in der frühen Sowjetunion, Göttingen, 2007.

WERTHEIMER Max, Produktives Denken, 2. Auflage, Frankfurt am Main, 1964.

WILSON Albert G., „Epilogue“, in F. Zwicky und ders. (Hg.), New Methods of Thought and Procedures, Contributions to the Symposium on Methodologies, Berlin et al., 1967, S. 333-338.

YATES Frances A., Gedächtnis und Erinnern. Mnemonik von Aristoteles bis Shakespeare, 6. Auflage, Berlin, 2001.

ZoBeL Dietmar und HARTMANN Rainer, Erfindungsmuster. TRIZ: Prinzipien, Analogien, Ordnungskriterien, Beispiele, Renningen, 2009.

ZWICKY Fritz, Morphology of Propulsive Power, Pasadena-Zürich, 1962.

_, Entdecken, Erfinden, Forschenim Morphologischen Weltbild, Taschenbuchausgabe, München, 1971.

-, Morphologische Forschung. Wesen und Wandel materieller und geistiger struktureller Zusammenhänge,

2. Auflage, Glarus, 1989.

-, Jeder ein Genie. Der berühmte Astrophysiker revolutioniert unsere Denkmethoden, 2. Auflage, BernGlarus, 1992.

\section{Internetlinks}

<http://www.hudson.org/index.cfm?fuseaction=HermanKahn> [Stand 05. Oktober 2011]. <http://www.mdr.de/damals/artikel84364.html> [Stand 05. Oktober 2011].

\section{Archive}

Nachlass Fritz Zwicky, Archiv der Landesbibliothek Glarus. 


\section{Abbildungsnachweise}

Abb. 1. - ZWICKY Fritz, Entdecken, Erfinden, Forschen im Morphologischen Weltbild, München, 1971, S. 130.

Abb. 2. - ZWicKY Fritz, Morphology of Propulsive Power, Pasadena-Zürich, 1962, S. 42.

Abb. 3. - NoRRIS Ken W., „The Morphological Approach to Engineering Design“, in J. C. Jones und D. G. Thornley (Hg.), Conference on Design Methods, New York, 1963, S. 115-140, hier S. 118.

Abb. 4. - AlTSCHULleR Genrich S., The Innovation Algorithm: TRIZ, Systematic innovation and technical creativity, Worcester, 2007, S. 268-269.

\section{NOTES}

1. Beide Begriffe sind für den zu untersuchenden Kontext geläufig und relevant, der Lesefreundlichkeit zuliebe wird im nachfolgenden Text jedoch in der Regel nur zusammenfassend von Kreativitätstechniken die Rede sein.

2. So kann etwa Ramon Llulls mittelalterliche Ars Magna et Ultima als Vorläufer heutiger algorithmischer Ideenfindungstechniken gelten, im Anschluss daran wäre an Leibniz' De arte combinatoria zu denken, in der vermittels deduktiver Methoden wie Kombinatorik oder Syllogistik verborgene wissenschaftliche Wahrheiten befördert werden sollten. Darüber hinaus werden freiere assoziative Verfahren wie die écriture automatique, die um die um 1900 sowohl in der Psychoanalyse als auch in Literatur und Kunst zum Einsatz kam, als Vorläufer heute bekannter ,professionalisierter' Kreativitätstechniken genannt. Vgl. ausführlich zu diesem Punkt: Mareis, 2011a, S. 203-241.

3. Publikation zur Beförderung von Kreativität in Psychologie, Management oder Pädagogik stammen im anglophonen Raum in der Zeit etwa von Crawford, 1954; Anderson, 1959; Getzels und Jackson, 1962; Gruber et al., 1964; Morgan, 1968. Aus dem deutschsprachigen Raum ab den 1970er Jahren: Kaufmann et al., 1972; Marr, 1973; Schiffler, 1973; Matussek, 1974; Bendixen, 1976; Preiser, 1976.

4. Kreativitätstechniken gelten beispielsweise als „Schlüsselkompetenzen“ für Studium und Beruf: Funke, 2008, S. 207-219.

5. Exemplarische für diesen Befund vgl. Schlicksupp, 1987; Strebel, 2007 oder Boos, 2010.

6. Zur Kulturgeschichte der Kybernetik siehe Hagner, Hörl, 2008. Zum Aspekt der Inter- und Antidisziplinarität kybernetischer Wissensproduktion siehe Pickering, Andrew, 2010, S. 3-15.

7. Zur Entstehungsgeschichte dieses militärisch-industriell-akademischen Verbundes siehe Leslie, 1993, S. 1-13.

8. Zum Aspekt der Angst im Kalten Krieg: Greiner, 2009, S. 19-21; zur Kulturgeschichte der Angst: Bourke, 2005.

9. Der Spiegel, 30. September 1974, S. 177. Siehe weiterführend: Matussek, 1974.

10. Cohen-Cole, 2009, S. 241.

11. Heubel, 2000, S. 7-8.

12. Horn, 2004, S. 314.

13. Zum Ablauf einer Brainstorming-Sitzung siehe Osborn, 1961, S. 101-109.

14. Zitat auf der Website des Hudson Institute: <http://www.hudson.org/index.cfm? fuseaction=HermanKahn>

15. $\mathrm{Zu}$ einigen kontroversen Stimmen siehe Pias, 2009, S. 5.

16. Zur RAND Corporation siehe Hounshell, 1997, S. 237-267. 
17. Pias, 2009, S. 6.

18. Aligica, Weinstein, 2009, S. 2.

19. Brandstetter, Pias, Vehlken, 2010, S. 25.

20. Der Spiegel, 3. April 1967, S. 123-140. Zit. nach: Pias, 2009, S. 9.

21. Brandstetter, Pias, Vehlken, 2010, S. 24-25.

22. Rowen, 2009, S. 99. Zit. nach Brandstetter, Pias, Vehlken, 2010, S. 39.

23. Brandstetter, Pias, Vehlken, 2010, S. 26.

24. Specht, 1960, S. 830 .

25. Robin, 2009, S. 18.

26. Zit. nach Jantsch, 1967, S. 133.

27. Jennings, 1949; Samelson, 1977. Zu einer Kritik der Army Mental Tests siehe Gould, 1988, S. 220-258.

28. Cohen-Cole, 2009, S. 241.

29. Matussek, 1974, S. 13.

30. Curtis, 1976, S. 10.

31. Guilford, 1950, S. 444-454. Nachfolgend zitiert nach der deutschen Übersetzung Guilford 1973.

32. Comrey, 1993, S. 202.

33. Guilford, 1973, S. 30.

34. Guilford, 1973, S. 27-29.

35. Guilford, 1973, S. 28.

36. Guilford, 1973, S. 26.

37. Bröckling, 2011, S. 91.

38. Guilford, 1973, S. 30

39. Cohen-Cole, 2009, S. 238.

40. Adorno, 1956.

41. Der Spiegel, 30. September 1974, S. 177.

42. Adorno et al., 1964. Zur Rezeptionsgeschichte der Studie: Samelson, 1986, S. 191-208.

43. Cohen Cole, 2009, S. 237.

44. Ulmann, 1973, S. 14

45. Bröckling, 2011, S. 91.

46. Cohen-Cole, 2009, S. 241.

47. Wertheimer, 1964 , S. 194-218.

48. Foucault, 1993 , S. 62.

49. Osborn, 1961, Cover.

50. Siehe ausführlicher zu Fritz Zwicky: Mareis, 2011b.

51. Zu seinen bedeutendsten Beiträgen zur Astrophysik und beobachtenden Astronomie siehe Straumann, Tammann, 2008.

52. Zu Leben und Werk von Fritz Zwicky siehe die umfangreiche Biographie von Müller, 1986 sowie Stöckli, Müller, 2008.

53. Müller, 1986, S. 338.

54. In seinen privaten Unterlagen finden sich durchaus Belege, dass Zwicky die zeitgenössische Kreativitätsforschung zur Kenntnis nahm. So sammelte er etwa einen Zeitungsartikel aus der Times vom 18. Februar 1957 zu Alex Osborns Brainstorming-Technik. Nachlass Fritz Zwicky, Archiv der Landesbibliothek Glarus, Dokumente Dossier FZH D 09.

55. Eine solche Einordnung wurde etwa durch Werner Hürlimann vorgenommen: Hürlimann, 1981.

56. Zwicky, 1971, S. 37.

57. Goethe, 1989, S. 12 .

58. Zwicky, 1971, S. 37.

59. Stöckli, 2008, S. 240. 
60. Zwicky, 1962, S. 5 .

61. Zwicky, 1989, aus dem Untertitel des Buches.

62. Zu den Jugendjahren von Fritz Zwicky siehe Stöckli, Müller, 2008, S. 12-31.

63. Müller, 1986, S. 50-53.

64. Zwicky, 1962, S. 17-20.

65. Siehe Zwicky, 1971, S. 1.

66. So wurde eine Vorlesungsreihe über die Morphologie an der ETH Zürich 1956 von Kollegen kontrovers diskutiert. Müller, 1986, S. 370-372.

67. Jantsch, 1967, S. 116

68. Zwicky, 1992, S. 235.

69. Zwicky, 1989, S. 18.

70. Zwicky, 1989, S. 18.

71. Stöckli, 2008, S. 227.

72. Weiterführende Literatur: Yates, 2001, S. 162-184; Badia, Bonner, 1988 sowie Bonner 1999.

73. Zwicky, 1989, S. 17f.

74. Stöckli, 2008, S. 231.

75. Wilson, 1967, S. 333-338.

76. Zwicky, 1971, S. 10.

77. Zwicky, 1971, S. 10.

78. Zwicky, 1971, S. 43f.

79. Zit. nach Müller, 1986, S. 196.

80. Müller, 1986, S. 196.

81. Zwicky, 1962, S. 17-20.

82. Zwicky, 1971, S. 11, Kursivsetzung im Original.

83. Zwicky, 1992, S. 76.

84. Zwicky, 1992, S. 100.

85. Curtis, 1976, S. 12.

86. Curtis, 1976, S.12. Die Autorin beklagte in diesem Zusammenhang zudem, dass psychoanalytische Ansätze in der Sowjetunion kaum rezipiert wurden.

87. Mehlhorn, 1984, S. 154.

88. Mehlhorn, 1984, S. 154.

89. Vöhringer, 2007, S. 14-15.

90. $\mathrm{Zu}$ GINKhUK und VchUTEMAS siehe Tillberg, 2003; zum Psychotechnischen Labor für Architektur in Moskau siehe Vöhringer, 2007.

91. Vöhringer, 2007, S. 10.

92. Vgl. Vöhringer, 2007, S. 77-84.

93. Grundlegend dazu Tillberg, 2003.

94. Rubinstein, 1962, S. 702.

95. Curtis, 1976, S. 40.

96. Curtis, 1976, S. 40.

97. Siehe zum Werk von Meilach: Schulz, 1976, S. 287-301.

98. Meilach, 1976, S. 261.

99. Meilach, 1976, S. 261.

100. Rubinstein, 1962, S. 703-723.

101. Rubinstein, 1962 , S. 703-704. Kursivsetzung im Original.

102. Rubinstein, 1962, S. 707.

103. Ulmann, 1973, S. 22.

104. Rubinstein, 1962, S. 705-706.

105. Altschuller, 1973, S. 91. 
106. Driesen, 2008, S. 120. Siehe als praktische Anwendungsbeispiele: Zobel, Hartmann, 2009; Koltze, Souchkov, 2011.

107. Altschuller, 1973, S. 43.

108. Lerner, 2007, S. 291.

109. Altschuller, 1998, S. 48.

110. Orloff, 2002, S. 34.

111. Altschuller, Seljuzki, 1983, S. 257.

112. Altschuller, 1973, S. 303-304.

113. Orloff, 2002, XI.

114. Altschuller, 1986, S. 25.

115. Altschuller, 1998, S. 48.

116. Altschuller, 1973, S. 8.

117. Altschuller, 1986, Vorwort der Originalausgabe, S. 6.

118. Altschuller, 1986, Vorwort der Originalausgabe, S. 7.

119. Altschuller, 1973, S. 13.

120. Zwicky, 1989, S. 18.

121. Altschuller, 1973, S. 13 (sic!).

122. Altschuller, Seljuzki, 1983, S. 251.

123. Altschuller, 1984.

124. Altschuller, 1986.

125. Altschuller, 1986, S. 28.

126. Altschuller, 1973, S. 113.

127. Altschuller, 1973, S. 113.

128. Vgl. beispielsweise Altschuller, 1973, S. 113-123.

129. Altschuller, 1973, S. 100-101.

130. Altschuller, 1986, S. 15-18.

131. Altschuller, 1998, S. 31.

132. Altschuller, 1986, $17 \mathrm{f}$.

133. Altschuller, 1973, S. 43.

134. Altschuller, 2007, S. 268-269.

135. Lerner, 2007, S. 291-292.

136. Lerner, 2007, S. 292-293.

137. Orloff, 2002, S. x.

138. Altschuller, Seljuzki, 1983, S. 7.

139. Altschuller, 1998, S. 39.

140. Thiel, 1998, S. 14, Kursivsetzung im Original.

141. Thiel, 1998, S. 13.

142. Mehlhorn, 1984, S. 161.

143. Guilford, 1973, S. 30.

144. Rubinstein, 1962, S. 707.

145. Altschuller, 1986, S. 32.

146. Altschuller, 1986, S. 32.

147. Altschuller, 1986, S. 148.

148. Altschuller, 1986, S. 148.

149. Altschuller, 1986, Vorwort zur 1. Auflage der deutschprachigen Ausgabe von Rainer Thiel, S. 4.

150. Lexikonbeitrag ,Neuererbewegung und Messe der Meister von Morgen', auf: < http:// www.mdr.de/damals/artikel84364.html>

151. Altschuller, 1973.

152. Driesen, 2008, S. 121. 
153. Rindfleisch, Thiel, 1994, S. 11.

154. Rindfleisch, Thiel, 1994, S. 15.

155. Rindfleisch, Thiel, 1994, S. 11.

156. Rindfleisch, Thiel, 1994, S. 15, siehe zu diesem Aspekt auch S. 20-23.

157. Rindfleisch, Thiel, 1994, S. 15.

158. <http://www.mdr.de/damals/artikel84364.html>

159. Ulbricht, 1969, S. 5.

160. <http://www.mdr.de/damals/artikel84364.html>

161. Vgl. zum Wettbewerbs- und Auszeichnungssystem in der Neuererbewegung: Stein, 1953, S. 20-24.

162. Als Vorbild für die Neuererbewegung diente unter anderen der Bergmann Alexej Stachanow, der am 31. August 1935 im Donez-Revier angeblich „spontan“ mit einem von ihm entwickelten neuartigen Presslufthammer ein Übersoll von 1400 \% erzeugt haben soll. Siehe dazu kritisch Stein, 1953, S. 7.

163. Rubinstein, 1962, S. 707.

164. Ulbricht, 1969, S. 5.

165. <http://www.mdr.de/damals/lexikon/artikel84364.html>

166. Stein, 1953, S. 5.

167. Stein, 1953, S. 17.

168. Rubinstein, 1962, 704, Kursivsetzung im Original.

169. Stein, 1953, S. 5.

170. Guilford, 1973, S. 43.

171. Heubel, 2000, S. 7.

172. Heubel, 2000, S. 7.

\section{RÉSUMÉS}

Kreativitäts- und Ideenfindungstechniken sind als rhetorische und methodische Konzepte längst ins kollektive Fachwissen vieler Berufs- und Wissenschaftsfelder sedimentiert, so etwa ins Design, in die Ingenieurswissenschaften, in die Künstlerische Forschung oder in die Pädagogik. Die zu beobachtende Popularität dieser Techniken in Kunst, Wissenschaft und Wirtschaft wird indes durch den Umstand kontrastiert, dass kaum differenzierte historiografische Studien existieren, die ihr gehäuftes Aufkommen und ihre Systematisierung während der Nachkriegszeit und des Kalten Krieges thematisieren. Während dieser Zeit wurden zahlreiche Kreativitäts- und Ideenfindungstechniken lanciert. Begleitet und geprägt wurden die Projekte zur Erforschung und Beförderung von Kreativität durch zeitgenössisch virulente Themen wie der Sputnik-Schock, die konstante atomare Bedrohung, oder die Debatte zu den Zwei-Kulturen der Wissenschaften (Snow, 1959). Im vorliegenden Text wird diese transnationale Dimension von Kreativitätstechniken exemplarisch anhand von erstens den so genannten Morphologischen Methoden und zweites der Erfindungstheorie TRIZ herausgearbeitet. Das erste Beispiel, die Morphologischen Methoden, gehen auf den aus der Schweiz stammenden Fritz Zwicky (1898-1974) zurück, der von 1942-1968 als Professor für Astrophysik am California Institute of Technology in Pasadena forschte und lehrte. Das zweite Beispiel behandelt die auf den russischen Ingenieur, Erfinder und Science-Fiction-Autor Genrich S. Altschuller (1926-1998) zurückgehende Theorie des erfinderischen Problemlösens (TRIZ). 
Die beiden genannten Kreativitätstechniken werden vorgestellt, kontextualisiert und miteinander in Beziehung gesetzt. Dabei sind vor allem solche Argumentationsmuster und Motive von Interesse, die auf zeit- und kulturspezifische Konzeptionen von Kreativität vor dem Hintergrund des Kalten Krieges verweisen.

Creativity and ideation techniques are being part of many occupational and scientific concepts and practises today. Techniques such as mind maps, brainstorming, so-called morphological methods, or complex invention algorithms as TRIZ (theory of inventive problem solving) can be found for instance within design, engineering, artistic research or educational science. Despite their popularity in practical application, from a scientific point of view, they often carry the stigma of the popular or pseudo-scientific. They are only rarely viewed as being historically conditioned - especially with regard to the post-war period and Cold War. During and directly following the end of World War II, one can observe that loads of techniques pop up for systematic stimulation, (re)discovery, and visualization of "ideas". The project of exploring and assisting creative processes and potentials was shaped by virulent contemporary occurrences and its debates, such as the Sputnik Crisis, the constant nuclear threat, or the debate on the two scientific cultures. (Snow, 1959). In my text I want to highlight this transnational dimension of creativity debates and techniques using the examples of first morphological methods, and second the invention algorithm TRIZ. Morphological methods were mainly developed and promoted by Swiss born astrophysicist Fritz Zwicky (1898-1974), professor at California Institute of Technology in Pasadena, after the end of World War II. In my second example I will introduce a theory of inventive problem solving called TRIZ, developed by the Russian engineer, inventor and author Genrich S. Altschuller (1926-1998). I will contextualize the two mentioned examples against the historical and cultural background of the post-war period and Cold War. As a result I will try to correlate them with regard to noticeable patterns and motives of argumentations referring to time and cultural specific conceptions of creativity, innovation and invention.

\section{INDEX}

Schlüsselwörter : Kalter Krieg, Morphologie, Fritz Zwicky, TRIS, Genrich Altschuller, Kreativitätstechniken, DDR, Neuererwesen

Mots-clés : Cold War, morphological methods, Fritz Zwicky, TRIZ, Guenrich Altshuller, creativity techniques, RDA, innovation

\section{AUTEUR}

\section{CLAUDIA MAREIS}

Universität Basel 